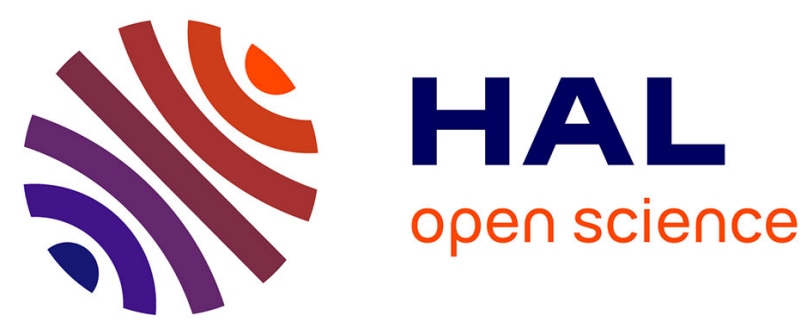

\title{
Multiple solutions to the Plateau problem for nonconstant mean curvature
}

\author{
Fabrice Bethuel, Olivier Rey
}

\section{To cite this version:}

Fabrice Bethuel, Olivier Rey. Multiple solutions to the Plateau problem for nonconstant mean curvature. Duke Mathematical Journal, 1994, 73 (3), pp.593-646. hal-00943475

\section{HAL Id: hal-00943475 \\ https://hal.science/hal-00943475}

Submitted on 27 Dec 2016

HAL is a multi-disciplinary open access archive for the deposit and dissemination of scientific research documents, whether they are published or not. The documents may come from teaching and research institutions in France or abroad, or from public or private research centers.
L'archive ouverte pluridisciplinaire HAL, est destinée au dépôt et à la diffusion de documents scientifiques de niveau recherche, publiés ou non, émanant des établissements d'enseignement et de recherche français ou étrangers, des laboratoires publics ou privés. 


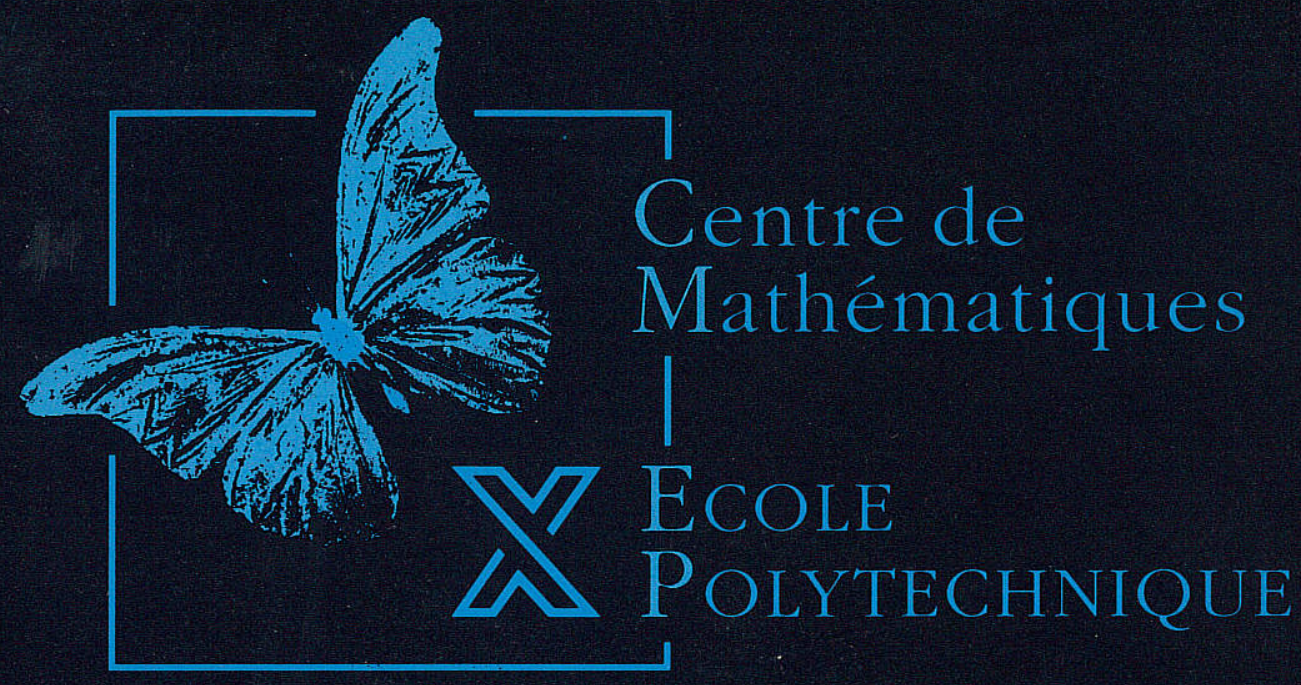

U.R.A. 169 du C.N.R.S.

Multiple solutions to the Plateau problem for nonconstant mean curvature
F. Bethuel
O. Rey

$\mathrm{N}^{\mathrm{o}} 1061$

juin 1993 


\section{MULTIPLE SOLUTIONS TO THE PLATEAU PROBLEM}

FOR NONCONSTANT MEAN CURVATURE

Fabrice Bethuel

ENPC-CERMA, La Courtine

93167 Noisy le Grand cedex, France.

CMLA Cachan, 61 avenue du Président Wilson 94235 Cachan cedex France.

Olivier Rey

Ecole Polytechnique, Centre de Mathématiques 91128 Palaiseau cedex France. 


\section{Introduction}

The questions that we are going to investigate originate from the classical socalled Plateau problem. $\Gamma$ being a Jordan curve in $\mathbf{R}^{3}$, the Plateau problem consists in finding disc-type surfaces of minimal area spanning $\Gamma$. Such a surface has mean curvature zero, and may be parametrized by a function

$$
u: D^{2}=\left\{(x, y) \in \mathbb{R}^{2} / x^{2}+y^{2}<1\right\} \rightarrow \mathbb{R}^{3}
$$

which satisfies

$$
\begin{gathered}
\Delta u=0 \text { in } D^{2} \\
\left|u_{x}\right|^{2}-\left|u_{y}\right|^{2}=u_{x} . u_{y}=0 \text { in } D^{2} \\
u_{\mid \partial D^{2}} \text { is a continuous monotone parametrization of } \Gamma .
\end{gathered}
$$

Conversely, a solution to (1.1) (1.2) (1.3) parametrizes, away from branch points, a surface with mean curvature zero spanning $\Gamma$, whose area is not necessarily minimal, but stationary.

In order to give this problem a variational structure, one often prefers to consider the related Dirichlet-type problem :

$$
\left\{\begin{aligned}
\Delta u=0 & \text { in } \quad D^{2} \\
u=\gamma & \text { on } \quad \partial D^{2}
\end{aligned}\right.
$$

where $\gamma$ is a given function from $\partial D^{2}$ to $\mathbb{R}^{3}$. Then, one can take advantage of the freedom that we have in the choice of $\gamma$ as a parametrization of $\Gamma$ to get the conformality condition (1.2) satisfied -see for instance [17] [18] [7] and references therein.

A solution to the classical Plateau problem having mean curvature zero, a natural generalization is to seek for surfaces spanning $\Gamma$ whose mean curvature is a given constant $H \in \mathbf{R}$. Equation (1.1) is then replaced by

$$
\Delta u=2 H u_{x} \wedge u_{y} \text { in } D^{2},
$$

and we denote by $(I I)$ the corresponding Dirichlet problem

$$
\left\{\begin{aligned}
\Delta u & =2 H u_{x} \wedge u_{y} \text { in } D^{2} \\
u & =\gamma \text { on } \partial D^{2} .
\end{aligned}\right.
$$


A further generalization is to look for surfaces whose mean curvature is prescribed, but not necessarily a constant. Namely, $H$ being a given function from $\mathbf{R}^{3}$ to $\mathbf{R}$, we impose the mean curvature of the surface at the point $u(x, y)$ to be $H(u(x, y))$. This leads to consider, instead of (1.1), the equation

$$
\Delta u=2 H(u) u_{x} \wedge u_{y} \quad \text { in } \quad D^{2},
$$

and the associated Dirichlet problem

$$
\left\{\begin{aligned}
\Delta u & =2 H(u) u_{x} \wedge u_{y} \text { in } D^{2} \\
u & =\gamma \text { on } \partial D^{2} .
\end{aligned}\right.
$$

Equation $(I I I)$ has a variational structure : solutions may be obtained as critical points of the functional $J$ defined by

$$
J_{H}(u)=E(u)+\frac{2}{3} \int_{D^{2}} Q(u) \cdot u_{x} \wedge u_{y}
$$

for $u \in H_{\gamma}^{1}\left(D^{2}, \mathbf{R}^{3}\right)=\left\{u \in H^{1}\left(D^{2}, \mathbf{R}^{3}\right)\right.$ s.t. $\left.u_{\mid \partial D^{2}}=\gamma\right\}$.

Here $E(u)$ denotes the Dirichlet integral

$$
E(u)=\frac{1}{2} \int_{D^{2}}|\nabla u|^{2}
$$

and $Q(u)$ is the following map from $\mathbf{R}^{3}$ to $\mathbf{R}^{3}$

$$
Q(u)=\left(\int_{0}^{u_{1}} H\left(t, u_{2}, u_{3}\right) d t, \int_{0}^{u_{2}} H\left(u_{1}, t, u_{3}\right) d t, \int_{0}^{u_{3}} H\left(u_{1}, u_{2}, t\right) d t\right) .
$$

Note that in case $H$ is a constant, $Q(u) \equiv H u$.

In 1969, Hildebrandt proved the existence of a first solution to (III), provided that the inequality

$$
\|\gamma\|_{L^{\infty}\left(\partial D^{2}, \mathbf{R}^{3}\right)}\|H\|_{L^{\infty}\left(D^{3}, \mathbf{R}^{3}\right)} \leq 1
$$

is satisfied [17]. From the observation of some special cases, Rellich had conjectured that for constant $H$, there should exist pairs of solutions. Such a conjecture was settled in 1984 by a result of Brezis and Coron [7]. Indeed, they proved that if (1.9) is strictly satisfied, there exists a second solution, as well for $(I I)$ as for the corresponding Plateau problem. However, their proof cannot be easily extended to the case of variable $H$. The main obstacle is to analyse the behaviour of the socalled Palais-Smale sequences, the functional $J$ being noncompact. To overcome this difficulty, Struwe introduced, as Sacks and Uhlenbeck in [28], a perturbed functional for which compactness properties hold. He was then able to prove in [37] a partial 
multiplicity result for variable curvatures close (in a special norm) to a constant. This result was slightly improved by Wang in [39].

In this paper, we focus on the existence of multiple solutions to $(I I I)$. We are then able to derive similar results concerning the Plateau problem. We propose a direct approach to the question, relying on both a careful analysis of Palais-Smale sequences and a priori bounds for solutions. This strategy allow us to prove the existence of a second solution to $(I I I)$, as soon as (1.9) is strictly satisfied, and $H$ is close to a constant in $L^{\infty}$-norm - see Theorem 4.2.

The paper is organized as follows :

- In Section 2, we recall the existence result of Hildebrandt, for the so-called "small solution".

- Section 3 is devoted to the result of Brezis and Coron concerning the existence of a second solution to (II).

- In Section 4, we develop the arguments which will make possible the proof of the main theorem, concerning the existence of a second solution to (III), provided $H$ is close enough to a constant.

- In Section 5, we the corresponding results that we can prove about the Plateau problem (1.5) (1.2) (1.3) - see Theorem 5.1.

- Lastly, we state in Section 6 some geometrical inequalities linking the mean curvature, the diameter and the area of a manifold, that the techniques that we used allow us to prove, or conjecture.

The main results of this paper were announced in [5] [6].

(*) We learn that some of the results presented here have been obtained independently and nearly at the same time by N. Jakobowsky.

\section{Acknowledgements}

We are indebted to J.M. Coron for bringing this problem to our attention and for numerous references in the litterature. We also thank and F. Hélein for stimulating discussions. 


\section{The "small solution".}

S. Hildebrandt proved in [17] the following result :

Theorem 2.1.- Let us assume that $\gamma \in H^{1 / 2}\left(\partial D^{2}, \mathbf{R}^{3}\right) \cap C^{0}\left(\partial D^{2}, \mathbf{R}^{3}\right), H \in$ $C^{1}\left(\mathbf{R}^{3}, \mathbf{R}^{3}\right)$, with

$$
\|\gamma\|_{L^{\infty}\left(\partial D^{2}, \mathbf{R}^{3}\right)}\|H\|_{L^{\infty}\left(D^{3}, \mathbf{R}^{3}\right)} \leq 1
$$

( $D^{n}$ denotes the unit ball of $\mathbf{R}^{n}$ ).

Problem (III) has a solution $\underline{u}$, which satisfies

$$
\|\underline{u}\|_{L^{\infty}\left(D^{2}, \mathbf{R}^{3}\right)} \leq\|\gamma\|_{L^{\infty}\left(\partial D^{2}, \mathbf{R}^{3}\right)} .
$$

\section{Remarks}

1. For the case $H \equiv 0$, see the pioneering works of Douglas [10] and Rado [24] [25] [26].

For the case $H \equiv$ constant, see the paper of Hildebrandt [18], and previous works by Heinz [15] and Werner [43]. Similar results have been obtained by Wente [40] [41] and Steffen [32] in case of constant $H$, by Steffen [32] for variable $H$.

The result of the theorem may also be found again by mean of the heat flow associated to the equation -see [27].

2. Using a minimizing procedure for the energy of the solutions of $(I I I)$ with respect to the parametrization $\gamma$ of $\Gamma$, one obtains a solution to the Plateau problem (1.5) (1.2) (1.3) -see [17].

3. The result is sharp when $\Gamma$ is a circle : Heinz proved in [16] that the problem had no solution for $H$ constant and $H .\|\gamma\|_{L^{\infty}}>1$.

4. $\underline{u} \in C^{0}\left(\bar{D}^{2}\right) \cap C^{2+\alpha}\left(D^{2}\right)$, for any $\left.\alpha \in\right] 0,1[$.

Proof of the theorem. We sketch the proof in the case $h=\|H\|_{L^{\infty}\left(D^{3}, \mathbf{R}^{3}\right)}<1$, $\|\gamma\|_{L^{\infty}\left(\partial D^{2}, \mathbf{R}^{3}\right)} \leq 1$, and $H$ smooth. The general case may be deduced using homogeneity arguments, an approximation method as in [17], and regularity results for elliptic systems [38].

Let $h^{\prime}$ be such that

$$
h<h^{\prime}<1
$$

and $\tilde{H}: \mathbf{R}^{3} \rightarrow \mathbf{R}^{3}$ a smooth function satisfying

$$
H \equiv \tilde{H} \quad \text { on } \quad D^{3} ; \tilde{H}(|u|)=0 \quad \text { for } \quad|u|>\frac{1}{h^{\prime}} ;\|\tilde{H}\|_{L^{\infty}\left(\mathbf{R}^{3}, \mathbf{R}^{3}\right)}<h^{\prime}
$$


We denote by $J_{\tilde{H}}$ and $\tilde{Q}$ the functionals corresponding to $\tilde{H}$ through (1.6) and (1.8) respectively. Note that we have

$$
\sup _{u \in \mathbf{R}^{3}}|\tilde{Q}(u)| \leq 1
$$

so that

$$
\frac{1}{3} E(u) \leq J_{\tilde{H}}(u) \leq \frac{5}{3} E(u) \quad \forall u \in H_{\gamma}^{1}\left(D^{2}, \mathbf{R}^{3}\right)
$$

It follows then from a result of Morrey [22] that the infimum of $\tilde{J}$ on $H_{\gamma}^{1}\left(D^{2}, \mathbf{R}^{3}\right)$ is achieved by a function $\underline{u}$ which is a solution to $(I I I)$ with $\tilde{H}$ instead of $H$. As we have also

$$
-\Delta|\underline{u}|^{2}=-2\left(|\nabla \underline{u}|^{2}+\underline{u} . \Delta \underline{u}\right) \leq-2|\nabla \underline{u}|^{2}(1-|\underline{u}||\tilde{H}(\underline{u})|) \leq 0,
$$

$\underline{u}$ is subharmonic in $D^{2}$, and the maximum principle yields

$$
|\underline{u}| \leq 1 \quad \text { in } \quad D^{2}
$$

$\tilde{H}$ being equal to $H$ on the unit ball of $\mathbb{R}^{3}, \underline{u}$ is a solution to $(I I I)$.

Now, we state a result which will be of fundamental importance in the sequel.

Lemma 2.2. - Suppose that $H_{0}$ is a constant, and

$$
\left|H_{0}\right| \cdot\|\gamma\|_{L^{\infty}\left(\partial D^{2}, \mathbf{R}^{3}\right)}<1
$$

The second derivative of $J_{H_{0}}$ at $\underline{u}$ is coercive. Namely, there exists $\delta>0$ such that

$$
J_{H_{0}}^{\prime \prime}(\underline{u})(\varphi, \varphi)=\int_{D^{2}}\left(|\nabla \varphi|^{2}+4 H_{0} \underline{u} \cdot \varphi_{x} \wedge \varphi_{y}\right) \geq \delta \int_{D^{2}}|\nabla \varphi|^{2}
$$

for any $\varphi \in H_{0}^{1}\left(D^{2}, \mathbf{R}^{3}\right)$.

For a proof of this lemma, see [7].

Remark. $\delta$ may be chosen independently of $\gamma$ as $\|\gamma\|_{L^{\infty}}$ goes to zero, $H_{0}$ being fixed. Indeed, $\|\underline{u}\|_{L^{\infty}} \leq\|\gamma\|_{L^{\infty}}$, so that

$$
J_{H_{0}}^{\prime \prime}(\underline{u})(\varphi, \varphi) \geq\left(1-2\left|H_{0}\right|\|\gamma\|_{L^{\infty}}\right) \int_{D^{2}}|\nabla \varphi|^{2} \geq \frac{1}{2} \int_{D^{2}}|\nabla \varphi|^{2}
$$

for $\|\gamma\|_{L^{\infty}}$ small enough. 


\section{The "large" solution for constant $H$.}

In order to prove the existence of a second solution to $(I I I)$, a natural idea is to take advantage of the characterization of the first solution as a minimum, to use some mountain pass-type procedure. This program was performed by Brezis and Coron in [7] for constant $H$, and Struwe gave another presentation of the result in [35]. Namely, it is proved in [7].

Theorem 3.1.- Let $\gamma \in H^{1 / 2}\left(\partial D^{2}, \mathbf{R}^{3}\right) \cap L^{\infty}\left(\partial D^{2}, \mathbf{R}^{3}\right)$.

Assume that $\gamma$ is not a constant on $\partial D^{2}$, and that $H_{0} \neq 0$ satisfies

$$
\left|H_{0}\right| \cdot\|\gamma\|_{L^{\infty}\left(\partial D^{2}, \mathbf{R}^{3}\right)}<1
$$

There exist at least two solutions of (III).

\section{Remarks}

1. If $\gamma$ is a constant, if follows from a result of Wente [42] that $u \equiv \gamma$ is the only solution.

2. For the existence of a second solution under different assumptions, see Struwe [34] and Steffen [33].

\subsection{The behaviour of Palais-Smale sequences.}

The main difficulty which one has to deal with concerning the functional $J_{H_{0}}$ is that the Palais-Smale condition (P.S.) is not satisfied. A Palais-Smale sequence for $J_{H_{0}}$ is a sequence $\left(u^{n}\right)$ such that

$$
\begin{gathered}
J_{H_{0}}\left(u^{n}\right) \text { is bounded } \\
J_{H_{0}}^{\prime}\left(u^{n}\right) \text { goes to zero in } H^{-1} .
\end{gathered}
$$

(P.S.) condition is said to be satisfied if and only if any sequence of this type is relatively compact.

Note that we have the following property :

Lemma 3.2.- Any Palais-Smale sequence is bounded in $H^{1}$. 
Proof. $J_{H_{0}}^{\prime}\left(u^{n}\right) \rightarrow 0$ in $H^{-1}$ writes

$$
\Delta u^{n}=2 H_{0} u_{x}^{n} \wedge u_{y}^{n}+f^{n}, f_{n} \rightarrow 0 \text { in } H^{-1} .
$$

Substracting from this equation the equality

$$
\Delta \underline{u}=2 H_{0} \underline{u}_{x} \wedge \underline{u}_{y},
$$

multiplying by $\varphi^{n}=u^{n}-\underline{u}$, and integrating on $D^{2}$, we obtain

$$
-\int_{D^{2}}\left|\nabla \varphi^{n}\right|^{2}=2 H_{0} \int_{D^{2}}\left(u_{x}^{n} \wedge u_{y}^{n}-\underline{u}_{x} \wedge \underline{u}_{y}\right) \varphi_{n}+\circ\left(\left\|\varphi^{n}\right\|_{H_{0}^{1}}\right) .
$$

Integration by parts yields

$$
-\int_{D^{2}}\left|\nabla \varphi^{n}\right|^{2}=4 H_{0} \int_{D^{2}} \underline{u} \cdot \varphi_{x}^{n} \wedge \varphi_{y}^{n}+2 H_{0} \int_{D^{2}} \varphi^{n} \cdot \varphi_{x}^{n} \wedge \varphi_{y}^{n}+\circ\left(\left\|\varphi^{n}\right\|_{H_{0}^{1}}\right)
$$

(see $[7$, Appendix $])$. On the other hand, we have

$$
J\left(u^{n}\right)=J(\underline{u})+\frac{1}{2} \int_{D^{2}}\left|\nabla \varphi^{n}\right|^{2}+2 H_{0} \int_{D^{2}} \underline{u} \cdot \varphi_{x}^{n} \wedge \varphi_{y}^{n}+\frac{2 H_{0}}{3} \int_{D^{2}} \varphi^{n} \cdot \varphi_{x}^{n} \wedge \varphi_{y}^{n}
$$

It follows from (3.1) (3.2) that

$$
\left|\int_{D^{2}} \varphi^{n} \cdot \varphi_{x}^{n} \wedge \varphi_{y}^{n}\right| \leq C+\circ\left(\left\|\varphi^{n}\right\|_{H_{0}^{1}}\right)
$$

Lemma 2.2 and (3.1) then show that $\left(\varphi^{n}\right)$ is bounded in $H_{0}^{1}$, and Lemma 3.2 follows.

From this we deduce that any Palais-Smale sequence is weakly relatively compact. It is easy to see that any weak limit is a solution to $(I I I)$. Indeed, observe that

$$
u_{x} \wedge u_{y}=\frac{1}{2}\left[\frac{\partial}{\partial x}\left(u \wedge u_{y}\right)+\frac{\partial}{\partial y}\left(u_{x} \wedge u\right)\right]
$$

Therefore, for any test function $\varphi \in C_{0}^{\infty}\left(D^{2}, \mathbf{R}^{3}\right)$

$$
\int_{D^{2}} u_{x} \wedge u_{y} \cdot \varphi=-\frac{1}{2} \int_{D^{2}} u \wedge u_{y} \cdot \varphi_{x}+u_{x} \wedge u \cdot \varphi_{y}
$$

and this last expression is continuous under weak convergence of $u$ in $H^{1}$.

However, the Palais-Smale sequences are not necessarily strongly relatively compact in $H^{1}$. Brezis and Coron have precisely analyzed this defect of convergence. Namely, it follows from [8]. 
Theorem 3.3. - Suppose that $\left(u^{n}\right)$ is a Palais-Smale sequence for $J_{H_{0}}$.

There exist

(i) $u^{0} \in H_{\gamma}^{1}$ solution to (III)

(ii) a finite number of nonconstant solutions $\omega_{1}, \cdots, \omega_{p}$ of $\Delta \omega=2 H_{0} \omega_{x} \wedge \omega_{y}$ on whole $\mathbf{R}^{2}$

(iii) sequences $\left(a_{1}^{n}\right) \cdots,\left(a_{p}^{n}\right)$ in $D^{2}$

(iv) sequences $\left(\varepsilon_{1}^{n}\right) \cdots,\left(\varepsilon_{p}^{n}\right)$ in $\mathbf{R}_{+}^{*}, \lim _{n \rightarrow+\infty} \varepsilon_{i}^{n}=0, \forall i$

such that, up to a subsequence, we have

$$
\left\|u^{n}-u^{0}-\sum_{i=1}^{p} \omega_{i}\left(\frac{\cdot-a_{i}^{n}}{\varepsilon_{i}^{n}}\right)\right\|_{H^{1}} \underset{n \rightarrow+\infty}{\rightarrow} 0
$$

and

$$
\begin{gathered}
\int_{D^{2}}\left|\nabla u^{n}\right|^{2}=\int_{D^{2}}\left|\nabla u^{0}\right|^{2}+\sum_{i=1}^{p} \int_{\mathbf{R}^{2}}\left|\nabla \omega_{i}\right|^{2}+\circ(1) \\
J_{H_{0}}\left(u^{n}\right)=J_{H_{0}}\left(u^{0}\right)+\sum_{i=1}^{p} J\left(\omega_{i}\right)+\circ(1)
\end{gathered}
$$

(with the straight forward definition of $J$ for maps from $\mathbb{R}^{2}$ to $\mathbf{R}^{3}$ ).

The proof of this theorem relies on a careful blow-up analysis inspired by the concentration-compactness method [21]. This result emphazises the role of solutions of the equation on whole $\mathbf{R}^{2}$. These solutions are completely classified, as stated in the following lemma [8].

Lemma 3.4.- Let $\omega \in L_{\text {loc }}^{1}\left(\mathbf{R}^{2}, \mathbf{R}^{3}\right)$ be such that

$$
\Delta \omega=2 H_{0} \omega_{x} \wedge \omega_{y} \quad \text { on } \quad \mathbf{R}^{2}, \int_{\mathbf{R}^{2}}|\nabla \omega|^{2}<+\infty \quad\left(H_{0} \neq 0\right)
$$

$\omega$ writes

$$
\omega(z)=\frac{1}{H} \Pi\left(\frac{P(z)}{Q(z)}\right)+C, z=(x, y)=x+i y
$$

where $\Pi: \mathbf{C} \rightarrow S^{2}$ denotes the stereographic projection, $P, Q$ are polynomials, and $C$ is a constant. In addition

$$
\int_{\mathbf{R}^{2}}|\nabla \omega|^{2}=\frac{8 \pi}{H_{0}^{2}} \max (\operatorname{deg} P, \operatorname{deg} Q)
$$

and

$$
J_{H_{0}}(\omega)=\frac{4 \pi}{3 H_{0}^{2}} \max (\operatorname{deg} P, \operatorname{deg} Q)
$$

(of course, the quotient $P / Q$ is supposed to be irreductible.) 


\subsection{The mountain-pass lemma}

We recall the mountain-pass lemma under its standard form.

Lemma.- Let $F$ be a $C^{1}$-functional on a Banach space $V$, which satisfies the following conditions:

(i) $F(0)=0$

(ii) $\exists r>0, \rho>0$ s.t. $F(v) \geq \rho, \forall v \in V,\|v\|=r$

(iii) $\exists v_{0} \in V,\left\|v_{0}\right\|>r$, s.t. $F\left(v_{0}\right)<0$

Setting

$$
c=\inf _{p \in P} \max _{t \in[0,1]} F(p((t))
$$

with $P=\left\{p \in C^{0}([0,1], V)\right.$ s.t. $\left.p(0)=0, p(1)=v_{0}\right\}$, there exists a sequence $\left(v^{n}\right)$ of $V$ such that

$$
\begin{aligned}
F\left(v^{n}\right) & \rightarrow c \\
F^{\prime}\left(v^{n}\right) & \rightarrow 0 \text { in } \quad V^{\prime}
\end{aligned}
$$

In case $F$ satisfies (P.S.), we infer the existence of a critical point at level $c$.

We apply this lemma to the functional $K: u \mapsto J_{H_{0}}(u+\underline{u})-J_{H_{0}}(\underline{u})$ on the space $V=H_{0}^{1}$.

Lemma 2.2 ensures that condition (ii) is satisfied. Moreover, condition (iii) is clearly satisfied, and the following lemma provides us with an upper bound on $c$.

Lemma 3.5.- We have

$$
c<\frac{4 \pi}{3 H^{2}}
$$

The proof of this estimate, which relies on careful computations along an explicit path, is given in [7]. 


\subsection{Construction of the large solution}

The mountain-pass lemma provides us with a Palais-Smale sequence $\left(u^{n}\right)$ for $J_{H_{0}}$. Up to a subsequence, $u^{n}$ converges weakly to a solution $u_{0}$ of $(I I)$. Moreover, using Theorem 3.3, we know that either

$$
u^{n} \rightarrow u_{0} \quad \text { in } H^{1}, \quad \text { and } \quad J_{H_{0}}\left(u_{0}\right)=J_{H_{0}}(\underline{u})+c
$$

or

$$
\begin{aligned}
J_{H_{0}}\left(u_{0}\right) & \leq J_{H_{0}}(\underline{u})+c-\inf \left\{J_{H_{0}}(\omega) / \Delta \omega=2 H \omega_{x} \wedge \omega_{y} \text { in } \mathbf{R}^{2}, \omega \not \equiv \text { constant }\right\} \\
& \leq J_{H_{0}}(\underline{u})+c-\frac{4 \pi}{3 H_{0}^{2}} \\
& <J_{H_{0}}(\underline{u})
\end{aligned}
$$

according to Lemma 3.5 .

Thus, in any case, $u_{0} \not \equiv \underline{u}$, and Theorem 3.1 follows.

Remark : In fact, it is possible to prove that we are in the first case, that is $\left(u_{n}\right)$ converges strongly to $u_{0}$.

Indeed, $\underline{u}$ is the solution to $(I I)$ with lowest energy. Let $u$ be another solution to the problem. Setting

$$
\varphi=u-\underline{u},
$$

We have

$$
\begin{aligned}
& J_{H_{0}}(u)=J_{H_{0}}(\underline{u}+\varphi)=J_{H_{0}}(\underline{u})+\frac{1}{2} J_{H_{0}}^{\prime \prime}(\underline{u})(\varphi, \varphi)+\frac{2 H_{0}}{3} \int_{D^{2}} \varphi \cdot \varphi_{x} \wedge \varphi_{y} \\
& J_{H_{0}}(\underline{u})=J_{H_{0}}(u-\varphi)=J_{H_{0}}(u)+\frac{1}{2} J_{H_{0}}^{\prime \prime}(u)(\varphi, \varphi)-\frac{2 H_{0}}{3} \int_{D^{2}} \varphi \cdot \varphi_{x} \wedge \varphi_{y}
\end{aligned}
$$

Summing these equalities, we obtain

$$
J_{H_{0}}^{\prime \prime}(\underline{u})(\varphi, \varphi)=-J_{H_{0}}^{\prime \prime}(u)(\varphi, \varphi)
$$

On the other hand, we have

$$
J_{H_{0}}^{\prime \prime}(u)(\varphi, \varphi)-J_{H_{0}}^{\prime \prime}(\underline{u})(\varphi, \varphi)=4 H_{0} \int_{D^{2}} \varphi \cdot \varphi_{x} \wedge \varphi_{y}
$$

Therefore

$$
J_{H_{0}}^{\prime \prime}(\underline{u})(\varphi, \varphi)=-2 H_{0} \int_{D^{2}} \varphi \cdot \varphi_{x} \wedge \varphi_{y}
$$

and we deduce from (3.4) and Lemma 2.2 that

$$
J_{H_{0}}(u)=J_{H_{0}}(\underline{u})+\frac{1}{6} J_{H_{0}}^{\prime \prime}(\underline{u})(\varphi, \varphi) \geq J_{H_{0}}(\underline{u})+\frac{\delta}{6} \int_{D^{2}}|\nabla \varphi|^{2} .
$$




\section{The second solution for variable $H$}

In this section, we no longer assume that $H$ is a constant. However, one might reasonably conjecture that Theorem 3.1. is still valid for variable $H$ "close" enough to a constant $H_{0} \neq 0$ which satisfies

$$
\left|H_{0}\right| \cdot\|\gamma\|_{L^{\infty}\left(\partial D^{2}, \mathbf{R}^{3}\right)}<1
$$

A natural approach to prove such a conjecture is to use a perturbation argument. Unfortunately, the analysis of the Palais-Smale sequences which was exposed in the previous section does not carry over easily to the case $H \not \equiv$ constant, even if $H$ is very close to $H_{0}$, whatever norm we may consider. There is a qualitative change as soon as $H$ is not exactly a constant. For instance, the argument that we used to prove that any weak limit of a Palais-Smale sequence is a solution to the equation see (3.3) - does not hold any more. Indeed, the nonlinear term is not continuous any more under weak convergence. In order to overcome this difficulty, Struwe, following an argument originally developped by Sacks and Uhlenbeck [28] in the context of harmonic maps between manifolds, introduced a perturbed functional, for which the Palais-Smale condition holds. For measuring the deviation of $H$ from a constant $H_{0}$, he considers the affine space of Lipschitz maps $H$ with

$$
\begin{gathered}
{\left[H-H_{0}\right]=\operatorname{ess} \sup _{u \in \mathbf{R}^{3}}\left[(1+|u|)\left(\left|H(u)-H_{0}\right|\right)+|\nabla H(u)|\right.} \\
\left.+\left|Q(u)-H_{0} u\right|+\left|d Q(u)-H_{0} i d\right|\right]<+\infty .
\end{gathered}
$$

He was then able to prove [37].

Theorem 4.1.- Assume that $H_{0} \in \mathbf{R}-\{0\}$ satisfies (4.1), and $\gamma \not \equiv$ constant. There exists a number $\alpha>0$ such that for a dense set of curvature functions $H$ in the $\alpha$-neighborhood of $H_{0}$, i.e. $\left[H-H_{0}\right]<\alpha,(I I I)$ admits at least two solutions.

This theorem was slightly improved by Wang, who showed that the same result is true for a whole neighborhood of $H_{0}$ in the (4.2)-norm [39].

Remark. In fact, Theorem 4.1 is proved just assuming that the functional $J_{H_{0}}$, corresponding to $H_{0}$, admits a strict relative minimizer in $H_{\gamma}^{1}$. Of course, according to Theorem 2.1 and Lemma 2.2, this is the case when (4.1) is satisfied. In the following, for sake of simplicity, we always assume that (4.1) holds. However, our method and results could easily been extended to the case where the only assumption is the existence a strict relative minimizer for $J_{H_{0}}$.

In this section, we propose a direct approach to the problem, which allows to prove the existence of a second solution with released assumptions on $H$. Namely, we prove : 
Theorem 4.2.- Assume that $H_{0} \in \mathbf{R}-\{0\}$ satisfies (4.1), and $\gamma \not \equiv$ constant.

There exists a number $\alpha>0$ such that for any curvature function $H \in C^{1}\left(\mathbf{R}^{3}, \mathbf{R}\right)$ which satisfies

$$
\left\|H-H_{0}\right\|_{L^{\infty}\left(\mathbf{R}^{3}, \mathbf{R}\right)}<\alpha
$$

(III) admits at least two solutions.

The idea to obtain the existence of a second solution is to use, as in the previous section, some mountain-pass procedure. However, the functional $J_{H}$ that we have to deal with is not $C^{1}$ any more on $H_{\gamma}^{1}$. In fact, it is not even obvious that

$$
J_{H}(u)=\frac{1}{2} \int_{D^{2}}|\nabla u|^{2}+\frac{2}{3} \int_{D^{2}} Q(u) \cdot u_{x} \wedge u_{y}
$$

is still defined on the whole space. Indeed, as soon as $H$ is not exactly a constant $H_{0}$, i.e. $Q(u) \not \equiv H_{0} u$, it is not clear that the last integral is convergent. However, as the integral

$$
\int_{D^{2}} u \cdot u_{x} \wedge u_{y}
$$

could be related to a volume, and then bounded in terms of the Dirichlet energy via some isoperimetric inequality, the same situation holds with $Q(u)$ instead of $u$. In this case, the integral

$$
\int_{D^{2}} Q(u) \cdot u_{x} \wedge u_{y}
$$

may be related to a volume, computed on some manifold equipped with the suitable metric, and still controlled in terms of Dirichlet integrals. (For more precisions, see $[32][4])$.

Once $J_{H}(u)$ is defined on $H_{\gamma}^{1}$, we have the formal expression

$$
J_{H}^{\prime}(u) \cdot \varphi=\int_{D^{2}} \nabla u \cdot \nabla \varphi+2 \int_{D^{2}} H(u) u_{x} \wedge u_{y} \cdot \varphi
$$

for any admissible test function $\varphi$. It is not possible to give a meaning to the last integral for general $u \in H_{\gamma}^{1}\left(D^{2}\right)$ and $\varphi \in H_{0}^{1}\left(D^{2}\right)$, and we have to consider a smaller space of admissible variations. For example, we can take $\varphi \in H_{0}^{1}\left(D^{2}\right) \cap L^{\infty}\left(D^{2}\right)$.

Thus, for any $u \in H_{\gamma}^{1}\left(D^{2}\right)$, we define $J_{H}^{\prime}(u)$ as the continuous linear map from $H_{0}^{1} \cap L^{\infty}$ to $\mathbf{R}$, which satisfies (4.3). We note that for any fixed $\varphi \in H_{0}^{1} \cap L^{\infty}$, the map $u \mapsto J_{H}^{\prime}(u) \cdot \varphi$ is continuous. We set

$$
\left\|J_{H}^{\prime}(u)\right\|=\sup _{\substack{\varphi \in H_{0}^{1} \cap L^{\infty} \\\|\varphi\|_{H_{0}^{1}}=1}}\left|J_{H}^{\prime}(u) \cdot \varphi\right|
$$


This norm may of course be infinite. If it is finite, there is a unique way to extend $J_{H}^{\prime}(u)$ to a continuous linear map from $H_{0}^{1}$ to $\mathbf{R}$, i.e. to an element of $H^{-1}$, since $H_{0}^{1}$ is complete and $H_{0}^{1} \cap L^{\infty}$ is dense in $H_{0}^{1}$.

Let us denote by $\underline{u}^{0}$ the small solution, given by Theorem 2.1, corresponding to $H_{0}$, and by $K_{H}$ the functional defined on $H_{\gamma}^{1}$

$$
K_{H}(u)=J_{H}\left(u+\underline{u}^{0}\right)-J_{H}\left(\underline{u}^{0}\right) .
$$

Suppose that $K_{H}$ satisfies the three conditions (i) (ii) (iii) of the mountain-pass lemma, i.e.

(i) $K_{H}(0)=0$

(ii) $\exists r>0, \rho>0$ s.t. $K_{H}(v) \geq \rho, \forall v \in H_{0}^{1},\|v\|_{H_{0}^{1}}=r$

(iii) $\exists v_{0} \in H_{0}^{1},\left\|v_{0}\right\|_{H_{0}^{1}}>r$ s.t. $K_{H}\left(v_{0}\right)<0$.

We set as usual

$$
c_{H}=\inf _{p \in P} \max _{t \in[0,1]} K_{H}(p(t))
$$

with

$$
P=\left\{p \in C^{0}\left([0,1], H_{0}^{1}\right) \text { s.t. } p(0)=0, p(1)=v_{0}\right\} .
$$

Let us assume now that there is no sequence $\left(v^{n}\right)$ in $H_{0}^{1}$ such that

$$
K_{H}\left(v^{n}\right) \rightarrow c_{H} ;\left\|K_{H}^{\prime}\left(v^{n}\right)\right\|=\left\|J_{H}^{\prime}\left(v^{n}+\underline{u}\right)\right\| \rightarrow 0,
$$

whence, by continuity, the existence of $\varepsilon>0, \eta>0$, such that

$$
\left\|K_{H}^{\prime}(v)\right\|>\eta, \forall v \in H_{0}^{1} \text { s.t. }\left|K_{H}(v)-c_{H}\right|<\varepsilon .
$$

The main point is that even if $K_{H}^{\prime}(v)$ may not be defined as an operator on the whole space $H_{0}^{1}$, its definition on the subset $H_{0}^{1} \cap L^{\infty}$ is sufficient to deform a path in $H_{\gamma}^{1}$. Namely, following the usual procedure to construct a pseudo-gradient, we get the existence of a locally Lipschitz continuous vector field $w(v) \in H_{0}^{1} \cap L^{\infty}$, defined on an open neighborhood $\mathcal{V}$ of $K_{H}^{-1}(] c_{H}-\varepsilon, c_{H}+\varepsilon[)$, such that for any $v \in \mathcal{V}$

$$
\begin{aligned}
& \|w(v)\|_{H_{0}^{1}} \leq 1 \\
& K_{H}^{\prime}(v) . w(v)<-\eta / 2
\end{aligned}
$$

Then, by mean of the flow associated to this vector field, we can push any path $p \in P$, linking 0 to $v_{0}$, such that

$$
\max _{t \in[0,1]} K_{H}(p(t))<c_{H}+\varepsilon
$$

under the level $c_{H}-\varepsilon$, that is deform it into a continuous path $p^{\prime}$, still linking 0 to $v_{0}$, and such that

$$
\max _{t \in[0,1]} K_{H}\left(p^{\prime}(t)\right) \leq c_{H}-\varepsilon
$$

in contradiction with the definition of $c_{H}$

The previous argument allows us to state : 
Lemma 4.3.- There exists a sequence $\left(u^{n}\right)$ in $H_{\gamma}^{1}$ such that

$$
\begin{gathered}
J_{H}\left(u^{n}\right) \rightarrow J_{H}\left(\underline{u}^{0}\right)+c_{H} \\
\left\|J_{H}^{\prime}\left(u^{n}\right)\right\| \rightarrow 0 .
\end{gathered}
$$

Proof. The only thing that remains to be verified is that conditions (i) (ii) (iii) are satisfied by $K_{H}$. We see that

(i) and (iii) are clearly satisfied.

(ii) will follow from Lemma 2.2. Let us introduce the quantity

$$
\beta_{H}=\sup _{u \in \mathbf{R}^{3}}\left[(1+|u|)\left|H(u)-H_{0}\right|+\left|Q(u)-H_{0} u\right|\right] .
$$

Assume that $\beta_{H}$ is finite. We have

$$
\left|J_{H}(u)-J_{H_{0}}(u)\right| \leq \frac{2}{3} \beta_{H} \int_{D^{2}}|\nabla u|^{2}, \quad \forall u \in H_{\gamma}^{1} .
$$

Hence

$$
\begin{gathered}
K_{H}(\varphi)=J_{H}\left(\underline{u}^{0}+\varphi\right)-J_{H}\left(\underline{u}^{0}\right) \\
\geq J_{H_{0}}\left(\underline{u}^{0}+\varphi\right)-J_{H_{0}}\left(\underline{u}^{0}\right)-\frac{2}{3} \beta_{H} \int_{D^{2}}\left|\nabla\left(\underline{u}^{0}+\varphi\right)\right|^{2}-\frac{2}{3} \beta_{H} \int_{D^{2}}\left|\nabla \underline{u}^{0}\right|^{2}
\end{gathered}
$$

According to Lemma 2.2, we know that

$$
J_{H_{0}}\left(\underline{u}^{0}+\varphi\right)-J_{H_{0}}\left(\underline{u}^{0}\right) \geq \frac{\delta}{2} \int_{D^{2}}|\nabla \varphi|^{2}
$$

for any $\varphi \in H_{0}^{1},\|\varphi\|_{H_{0}^{1}}$ small enough. Consequently, there exist $r, \rho>0$ such that

$$
K_{H}(\varphi) \geq \rho, \forall \varphi \in H_{0}^{1},\|\varphi\|_{H_{0}^{1}}=r
$$

provided that $\beta_{H}$ is small enough.

Let us now give the proof of Theorem 4.2, with this transitory additional assumption on the finiteness and the smallness of $\beta_{H}$. A priori estimates on the $L^{\infty}$-norm of solutions to our problem will then give us the full conclusion.

Once we got, as in the previous section, a Palais-Smale sequence as stated in lemma 4.3, we have to show that such a sequence is bounded in $H^{1}$. Namely, we prove : 
Lemma 4.4.- Any sequence $\left(u^{n}\right)$ in $H_{\gamma}^{1}$ which satisfies

$$
J_{H}\left(u^{n}\right) \text { bounded } ;\left\|J_{H}^{\prime}\left(u^{n}\right)\right\| \text { goes to zero }
$$

is bounded in $H^{1}$, provided that $\beta_{H}$ is small enough.

Proof.

The argument is the same as for Lemma 3.2. $\left\|J_{H}^{\prime}\left(u^{n}\right)\right\| \rightarrow 0$ means that

$$
\Delta u^{n}=2 H\left(u^{n}\right) u_{x}^{n} \wedge u_{y}^{n}+f^{n} \quad \text { in } \quad D^{2},
$$

with $f^{n} \rightarrow 0$ in $H^{-1}$. We multiply this equality by $\varphi^{n}=u^{n}-\underline{u}^{0}$, and integrate on $D^{2}$. Note that this is possible, since $\underline{u}^{0} \in L^{\infty}$, and we assumed that $\left|H(u) u-H_{0} u\right|$ is bounded for $u \in \mathbb{R}^{3}$. We obtain

$\int_{D^{2}}\left[-\Delta \varphi^{n}+2 H_{0}\left(u_{x}^{n} \wedge u_{y}^{n}-\underline{u}_{x}^{0} \wedge \underline{u}_{y}^{0}\right)\right] \cdot \varphi^{n}+2\left(H\left(u^{n}\right)-H_{0}\right) u_{x}^{n} \wedge u_{y}^{n} \cdot \varphi^{n}=o\left(\left\|\varphi^{n}\right\|_{H_{0}^{1}}\right)$

where each term is well defined. This yields

$$
\int_{D^{2}}\left[-\Delta \varphi^{n}+2 H_{0}\left(u_{x}^{n} \wedge u_{y}^{n}-\underline{u}_{x}^{0} \wedge \underline{u}_{y}^{0}\right)\right] \cdot \varphi^{n}=o\left(\left\|\varphi^{n}\right\|_{H_{0}^{1}}\right)+0\left(\beta_{H}\left\|\varphi^{n}\right\|_{H_{0}^{1}}^{2}\right) .
$$

Integrations by parts give (see [7, Appendix])

$$
\begin{gathered}
\int_{D^{2}}\left|\nabla \varphi^{n}\right|^{2}+4 H_{0} \int_{D^{2}} \underline{u}^{0} \cdot \varphi_{x}^{n} \wedge \varphi_{y}^{n}+2 H_{0} \int_{D^{2}} \varphi^{n} \cdot \varphi_{x}^{n} \wedge \varphi_{y}^{n} \\
=o\left(\left\|\varphi^{n}\right\|_{H_{0}^{1}}\right)+0\left(\beta_{H}\left\|\varphi^{n}\right\|_{H_{0}^{1}}^{2}\right) .
\end{gathered}
$$

On the other hand, we have

$$
\begin{aligned}
J_{H}\left(u^{n}\right) & =J_{H}\left(\underline{u}^{0}+\varphi^{n}\right) \\
& =\frac{1}{2} \int_{D^{2}}\left|\nabla\left(\underline{u}^{0}+\varphi^{n}\right)\right|^{2}+\frac{2}{3} \int_{D^{2}} Q\left(\underline{u}^{0}+\varphi^{n}\right) \cdot\left(\underline{u}^{0}+\varphi^{n}\right)_{x} \wedge\left(\underline{u}^{0}+\varphi^{n}\right)_{y} \\
& =\frac{1}{2} \int_{D^{2}}\left|\nabla \underline{u}^{0}\right|^{2}+\frac{1}{2} \int_{D^{2}}\left|\nabla \varphi^{n}\right|^{2}+\int_{D^{2}} \nabla \underline{u} \cdot \nabla \varphi^{n} \\
& +\frac{2}{3} H_{0} \int_{D^{2}}\left(\underline{u}^{0}+\varphi^{n}\right) \cdot\left(\underline{u}^{0}+\varphi^{n}\right)_{x} \wedge\left(\underline{u}^{0}+\varphi^{n}\right)_{y}+0\left[\beta_{H}\left(1+\left\|\varphi^{n}\right\|_{H_{0}^{1}}^{2}\right)\right] \\
& =J_{H}\left(\underline{u}^{0}\right)+\frac{1}{2} \int_{D^{2}}\left|\nabla \varphi^{n}\right|^{2}-2 H_{0} \int_{D^{2}} \varphi^{n} \cdot \underline{u}_{x}^{0} \wedge \underline{u}_{y}^{0} \\
& +\frac{2}{3} H_{0} \int_{D^{2}} \underline{u}^{0} \cdot\left(\varphi_{x}^{n} \wedge \underline{u}_{y}^{0}+\underline{u}_{x}^{0} \wedge \varphi_{y}^{n}+\varphi_{x}^{n} \wedge \varphi_{y}^{n}\right)+\frac{2}{3} H_{0} \int_{D^{2}} \varphi^{n} \cdot\left(\underline{u}^{0}+\varphi^{n}\right)_{x} \wedge\left(\underline{u}^{0}+\varphi^{n}\right)_{y} \\
& +0\left[\beta_{H}\left(1+\left\|\varphi^{n}\right\|_{H_{0}^{1}}^{2}\right)\right]
\end{aligned}
$$


Integrating by parts, we obtain

$$
\begin{gathered}
J_{H}\left(u^{n}\right)=J_{H}\left(\underline{u}^{0}\right)+\frac{1}{2} \int_{D^{2}}\left|\nabla \varphi^{n}\right|^{2}+2 H_{0} \int_{D^{2}} \underline{u}^{0} \cdot \varphi_{x}^{n} \wedge \varphi_{y}^{n} \\
+\frac{2}{3} H_{0} \int_{D^{2}} \varphi^{n} \cdot \varphi_{x}^{n} \wedge \varphi_{y}^{n}+0\left[\beta_{H}\left(1+\left\|\varphi^{n}\right\|_{H_{0}^{1}}^{2}\right)\right]
\end{gathered}
$$

Comparing (4.13) and (4.14), the boundedness of $J_{H}\left(u^{n}\right)$ yields

$$
\left|\int_{D^{2}} \varphi^{n} \cdot \varphi_{x}^{n} \wedge \varphi_{y}^{n}\right| \leq C+0\left[\beta_{H}\left(1+\left\|\varphi^{n}\right\|_{H_{0}^{1}}^{2}\right)\right]+o\left(\left\|\varphi^{n}\right\|_{H_{0}^{1}}\right)
$$

Then, (4.14) and Lemma 2.2 imply that $\left(\varphi^{n}\right)$ is bounded in $H_{0}^{1}$, provided that $\beta_{H}$ is small enough, and Lemma 4.4. follows.

What we need at this step, in order to give a complete description of the situation, is a precise analysis of the behaviour of the Palais-Smale sequences. Since such a sequence is bounded, according to Lemma 4.4 , it is weakly relatively compact. Up to a subsequence, we can assume that $\left(u^{n}\right)$ converges weakly in $H^{1}$ to some limit $u$. However, the nonlinear term of the equation is not continuous any more under weak convergence, and new arguments have to be developped, in order to prove that $u$, weak limit of a Palais-Smale sequence, is a solution to the problem. This result was established in [3], i.e.

Theorem 4.5.- Assume that $H: \mathbf{R}^{3} \rightarrow \mathbf{R}$ satisfies

$$
\sup _{u \in \mathbf{R}^{3}}|\nabla H(u)|<+\infty .
$$

Let $\left(u^{n}\right)$ be a bounded sequence in $H_{\gamma}^{1}$ such that

$$
\Delta u^{n}=2 H\left(u^{n}\right) u_{x}^{n} \wedge u_{y}^{n}+f^{n} \quad \text { in } \quad D^{2}
$$

with $f^{n} \rightarrow 0$ in $H^{-1}$.

Any weak limit $u$ of a subsequence of $\left(u^{n}\right)$ satisfies

$$
\Delta u=2 H(u) u_{x} \wedge u_{y} \quad \text { in } \quad D^{2} .
$$

We are now able to prove an equivalent to Theorem 3.3 for variable $H$, characterizing the lack of compactness of Palais-Smale sequences. The statement reads as : 
Theorem 4.6.- Assume that $H: \mathbf{R}^{3} \rightarrow \mathbf{R}$ satisfies

$$
\sup _{u \in \mathbf{R}^{3}}(1+|u|)|\nabla H(u)|<+\infty
$$

Let $\left(u^{n}\right)$ be a bounded Palais-Smale sequence for $J_{H}$. Then there exist

(i) $u^{0} \in H_{\gamma}^{1}$, solution to (III)

(ii) a finite number of nonconstant solutions $\omega_{1}, \cdots, \omega_{p}$ of $\Delta \omega=2 H(\omega) \omega_{x} \wedge \omega_{y}$ on whole $\mathbf{R}^{2}$

(iii) sequences $\left(a_{1}^{n}\right), \cdots,\left(a_{p}^{n}\right)$ in $D^{2}$

(iv) sequences $\left(\varepsilon_{1}^{n}\right), \cdots,\left(\varepsilon_{p}^{n}\right)$ in $\mathbf{R}_{+}^{*}, \lim _{n \rightarrow+\infty} \varepsilon_{i}^{n}=0, \forall i$

such that, up to a subsequence, we have

$$
\left\|u^{n}-u^{0}-\sum_{i=1}^{p} \omega_{i}\left(\frac{-a_{i}^{n}}{\varepsilon_{i}^{n}}\right)\right\|_{H^{1}} \underset{u \rightarrow+\infty}{\longrightarrow} 0
$$

and

$$
\begin{gathered}
\int_{D^{2}}\left|\nabla u^{n}\right|^{2}=\int_{D^{2}}\left|\nabla u^{0}\right|^{2}+\sum_{i=1}^{p} \int_{\mathbf{R}^{2}}\left|\nabla \omega^{i}\right|^{2}+o(1) \\
J_{H}\left(u^{n}\right)=J_{H}\left(u^{0}\right)+\sum_{i=1}^{p} J_{H}\left(\omega_{i}\right)+o(1) .
\end{gathered}
$$

This theorem is a consequence of the more general Theorem 5.6 below, the proof of which, relying on a careful blow up analysis, is given in Appendix.

We do not have at our disposal an equivalent to Lemma 3.4, describing exactly the set of solutions to the equation $\Delta \omega=2 H(\omega) \omega_{x} \wedge \omega_{y}$ on the whole space, with finite energy. However, we have the useful estimate :

Lemma 4.7.- Let $\omega \in L_{\text {loc }}^{1}\left(\mathbf{R}^{2}, \mathbf{R}^{3}\right)$ be such that

$$
\Delta \omega=2 H(\omega) \omega_{x} \wedge \omega_{y} \quad \text { on } \quad \mathbf{R}^{2}, \omega \not \equiv \text { constant, } \int_{\mathbf{R}^{2}}|\nabla \omega|^{2}<+\infty
$$

Then

$$
J_{H}(\omega):=\frac{1}{2} \int_{\mathbf{R}^{2}}|\nabla \omega|^{2}+\frac{2}{3} \int_{\mathbf{R}^{2}} Q(\omega) \cdot \omega_{x} \wedge \omega_{y} \geq \frac{4 \pi}{3 H_{0}^{2}}+0\left(\beta_{H}\right) .
$$

Proof. First note that $u \in L_{\text {loc }}^{1}\left(\mathbf{R}^{2}, \mathbf{R}^{3}\right)$ and $\int_{\mathbf{R}^{2}}|\nabla \omega|^{2}<+\infty$ implies that $u \in$ $H^{1}\left(\Omega, \mathbf{R}^{3}\right)$ for any bounded $\Omega \subset \mathbf{R}^{2}$. Then, if $H \in C^{1}\left(\mathbf{R}^{3}, \mathbf{R}\right)$ with bounded gradient,

$$
\omega \in C^{2+\mu}\left(\mathbf{R}^{2}, \mathbf{R}^{3}\right), \forall \mu \in(0,1)
$$


- see [13] [2]. For $z=x+i y$, the function

$$
\phi(z)=\left(\left|\omega_{x}\right|^{2}-\left|\omega_{y}\right|^{2}\right)+2 i \omega_{x} \cdot \omega_{y}
$$

is holomorphic in $\mathbf{R}^{2}$. Moreover, $\phi \in L^{1}\left(\mathbf{R}^{2}\right)$, since $\int_{\mathbf{R}^{2}}|\nabla \omega|^{2}$ is assumed to be finite. Therefore, $\phi$ has to be identically zero, and $\omega$ is conformal. As a consequence, $\omega$ is bounded on $\mathbb{R}^{2}$ - see the proof of Theorem 4.8 below. This implies that

$$
-\int_{\mathbf{R}^{2}}|\nabla \omega|^{2}=2 \int_{\mathbf{R}^{2}} H(\omega) \omega \cdot \omega_{x} \wedge \omega_{y} .
$$

Indeed, as in [23], let $\psi \in C_{0}^{\infty}\left(\mathbf{R}^{2}, \mathbf{R}\right)$ be such that

$$
\psi(z)=1 \text { for }|z|<\frac{1}{2} ; \psi_{(z)}=0 \text { for }|z|>1
$$

and

$$
\psi_{n}(z)=\psi(z / n)
$$

We multiply the equation $\Delta \omega=2 H \omega_{x} \wedge \omega_{y}$ by $\psi_{n} \omega$, and integrate over $\mathbb{R}^{2}$. We obtain

$$
\int_{\mathbf{R}^{2}} \psi_{n} \omega \cdot \Delta \omega=-\int_{\mathbf{R}^{2}} \psi_{n}|\nabla \omega|^{2}-\int_{\mathbf{R}^{2}} \nabla \psi_{n} \cdot \omega \nabla \omega=2 \int_{\mathbf{R}^{2}} \psi_{n} H(\omega) \omega \cdot \omega_{x} \wedge \omega_{y} .
$$

Remark that

$$
\left|\int_{\mathbf{R}^{2}} \nabla \psi_{n} \cdot \omega \nabla \omega\right| \leq\|\omega\|_{L^{\infty}}\left(\int_{\mathbf{R}^{2}}\left|\nabla \psi_{n}\right|^{2}\right)^{1 / 2}\left(\int_{n / 2 \leq|z| \leq n}|\nabla \omega|^{2}\right)^{1 / 2}
$$

Since $\int_{\mathbf{R}^{2}}\left|\nabla \psi_{n}\right|^{2}=\int_{\mathbf{R}^{2}}|\nabla \psi|^{2},|\nabla \omega|^{2} \in L^{1}$, and $\omega \in L^{\infty}$, Lebesgue's theorem yields (4.15). Hence the estimates

$$
\begin{aligned}
& \left.\left|\int_{\mathbf{R}^{2}}\right| \nabla \omega\right|^{2}+\left.2 \int_{\mathbf{R}^{2}} Q(\omega) \cdot \omega_{x} \wedge \omega_{y}\left|\leq \beta_{H} \int_{\mathbf{R}^{2}}\right| \nabla \omega\right|^{2} \\
& \left.\left|\int_{\mathbf{R}^{2}}\right| \nabla \omega\right|^{2}+\left.2 H_{0} \int_{\mathbf{R}^{2}} \omega \cdot \omega_{x} \wedge \omega_{y}\left|\leq \beta_{H} \int_{\mathbf{R}}\right| \nabla \omega\right|^{2}
\end{aligned}
$$

For any $\varphi \in H_{0}^{1}\left(\Omega, \mathbf{R}^{3}\right)$, where $\Omega$ is a bounded domain in $\mathbf{R}^{2}$, we have the isoperimetric inequality [40]

$$
\left|\int_{\Omega} \varphi \cdot \varphi_{x} \wedge \varphi_{y}\right|^{2 / 3} \leq \frac{1}{(32 \pi)^{1 / 3}} \int_{\Omega}|\nabla \varphi|^{2}
$$

Applying this to $\psi_{n} \omega$, Lebesgue's theorem proves that

$$
\left|\int_{\mathbf{R}^{2}} \omega \cdot \omega_{x} \wedge \omega_{y}\right|^{2 / 3} \leq \frac{1}{(32 \pi)^{1 / 3}} \int_{\mathbf{R}^{2}}|\nabla \omega|^{2}
$$


Thus, (4.7) implies that

$$
\int_{\mathbf{R}^{2}}|\nabla \omega|^{2} \geq \frac{8 \pi}{H_{0}^{2}}-\beta_{H}
$$

On the other hand, according to (4.16), we have

$$
J_{H}(\omega) \geq\left(\frac{1}{6}-\beta_{H}\right) \int_{\mathbf{R}^{2}}|\nabla \omega|^{2},
$$

hence the result follows.

Let now $\eta$ be some strictly positive number, such that

$$
\begin{gathered}
2 \eta \leq c_{H} \leq \frac{4 \pi}{3 H_{0}^{2}}-3 \eta \\
\inf \left\{J_{H}(\omega) / \Delta \omega=2 H(\omega) \omega_{x} \wedge \omega_{y} \text { on } \mathbf{R}^{2}, \omega \neq \equiv \text { constant, } \int_{\mathbf{R}^{2}}|\nabla \omega|^{2}<+\infty\right\} \\
>\frac{4 \Pi}{3 H_{0}^{2}}-\eta .
\end{gathered}
$$

The existence of such an $\eta$ follows directly from Lemmas 3.5 and 4.7 , as soon as $\beta_{H}$ is small enough.

Lemma 4.3 provides us with a Palais-Smale sequence $\left(u^{n}\right)$ for $J_{H}$, with

$$
J_{H}\left(u^{n}\right) \rightarrow J_{H}\left(\underline{u}^{0}\right)+c_{H} \quad \text { as } \quad n \rightarrow+\infty .
$$

Moreover, assuming that $\beta_{H}$ is small enough, this sequence is bounded in $H^{1}$, as Lemma 4.4 shows. Therefore, assuming that (*) holds, we can apply Theorem 4.6. As a consequence, we know that up to a subsequence, $\left(u^{n}\right)$ converges weakly to a solution $\bar{u}$ to $(I I I)$, and either

$$
J_{H}(\bar{u})=J_{H}\left(\underline{u}^{0}\right)+c_{H}
$$

or

$$
\begin{gathered}
J_{H}(\bar{u}) \leq J_{H}\left(\underline{u}^{0}\right)+c_{H} \\
-\inf \left\{J_{H}(\omega) / \Delta \omega=2 H(\omega) \omega_{x} \wedge \omega_{y} \text { on } \mathbf{R}^{2}, \omega \neq \equiv \text { constant }, \int_{\mathbf{R}^{2}}|\nabla \omega|^{2}<+\infty\right\}
\end{gathered}
$$

Note that $\underline{u}^{0}$ and $\underline{u}$ realizing respectively the infimum of $J_{H_{0}}$ and $J_{H}$ on a bounded subset of $H_{\gamma}^{1}$, it follows from (4.11) that

$$
\left|J_{H}\left(\underline{u}^{0}\right)-J_{H}(\underline{u})\right|<\eta
$$

for $\beta_{H}$ small enough. 
Comparing (4.18) (4.19) and (4.20), we see that either

$$
J_{H}(\bar{u}) \geq J_{H}(\underline{u})+\eta
$$

or

$$
J_{H}(\bar{u}) \leq J_{H}(\underline{u})-\eta .
$$

In any case, $\bar{u} \not \equiv \underline{u}$, and we have a second solution to $(I I I)$, provided that $(*)$ is satisfied and $\beta_{H}$ is small enough.

In fact, we are able to prove that we are in the first case, i.e. the convergence of $\left(u^{n}\right)$ to $\bar{u}$ is strong. The argument is the same as in Section 3. Setting $\varphi=\bar{u}-\underline{u}$, we have

$$
\begin{aligned}
J_{H}(\bar{u}) & =J_{H}(\underline{u}+\varphi) \\
& =J_{H_{0}}(\underline{u}+\varphi)+o(1) \\
& =J_{H_{0}}(\underline{u})+\frac{1}{2} \int_{D^{2}}|\nabla \varphi|^{2}+2 H_{0} \int_{D^{2}} \underline{u} \cdot \varphi_{x} \wedge \varphi_{y}+\frac{2}{3} H_{0} \int_{D^{2}} \varphi \cdot \varphi_{x} \wedge \varphi_{y}+o(1)
\end{aligned}
$$

as $\beta_{H} \rightarrow 0$. (Note that Lemma 4.4 provides us with a $H^{1}$-bound for $\bar{u}$, independent of $\beta_{H}$ as soon as $\beta_{H}$ is small enough. Then we use (4.11)). We write also

$$
\begin{aligned}
J_{H}(\underline{u}) & =J_{H}(\bar{u}-\varphi) \\
& =J_{H_{0}}(\bar{u}-\varphi)+o(1) \\
& =J_{H_{0}}(\bar{u})+\frac{1}{2} \int_{D^{2}}|\nabla \varphi|^{2}+2 H_{0} \int_{D^{2}} \bar{u} \cdot \varphi_{x} \wedge \varphi_{y}-\frac{2}{3} H_{0} \int_{D^{2}} \varphi \cdot \varphi_{x} \wedge \varphi_{y}+o(1) .
\end{aligned}
$$

Summing these equalities, we obtain, taking account of (4.11)

$$
\int_{D^{2}}|\nabla \varphi|^{2}+4 H_{0} \int_{D^{2}} \underline{u} \cdot \varphi_{x} \wedge \varphi_{y}+2 H_{0} \int_{D^{2}} \varphi \cdot \varphi_{x} \wedge \varphi_{y}=o(1)
$$

so that we have

$$
J_{H}(\bar{u})=J_{H_{0}}(\underline{u})+\frac{1}{6}\left(\int_{D^{2}}|\nabla \varphi|^{2}+4 H_{0} \int_{D^{2}} \underline{u} \cdot \varphi_{x} \wedge \varphi_{y}\right)+o(1) .
$$

As $\underline{u}$ goes to $\underline{u}^{0}$ as $\beta_{H}$ goes to zero, (4.11) and Lemma 2.2 yield

$$
J_{H}(\bar{u}) \geq J_{H}(\underline{u})+\frac{\delta}{6} \int_{D^{2}}|\nabla \varphi|^{2}+o(1)
$$

hence the conclusion follows.

The last ingredient that we need, to complete the proof of Theorem 4.2 , is an a priori bound for the $L^{\infty}$-norm of the solutions in terms of the $H^{1}$-norm. Namely, we prove : 
Theorem 4.8. - Assume that $H: \mathbf{R}^{3} \rightarrow \mathbf{R}$ is smooth and bounded on $\mathbf{R}^{3}$. Let $u$ be a smooth solution to (III). There exist $C>0$, depending on the $L^{\infty}$-norm of $H$ only, such that

$$
\|u\|_{L^{\infty}} \leq\|\gamma\|_{L^{\infty}}+C\left(\int_{D^{2}}|\nabla u|^{2}+1\right)
$$

Proof of Theorem 4.2 completed. Let $\beta_{0}$ be such that, for any $H \in C^{1}\left(\mathbf{R}^{3}, \mathbf{R}\right)$ close to $H_{0}$, with

$$
\beta_{H}<\beta_{0}
$$

and $(*)$ satisfied, there exists, according to the previous argument, a second solution $\bar{u}$ to $(I I I)$. Lemma 4.4 provides us with a $H^{1}$-bound for $\bar{u}$, namely

$$
\|\bar{u}\|_{H^{1}}<R
$$

with $R$ independent of $H$ satisfying (*) and (4.24). Then, Theorem 4.8 yields

$$
\|\bar{u}\|_{L^{\infty}} \leq\|\gamma\|_{L^{\infty}}+C\left(R^{2}+1\right)=R^{\prime}
$$

There exists $\alpha>0$ such that, for any $H \in C^{1}\left(\mathbf{R}^{3}, \mathbf{R}\right)$ satisfying

$$
\left\|H-H_{0}\right\|_{L^{\infty}}<\alpha
$$

one can find $\tilde{H} \in C^{1}\left(\mathbf{R}^{3}, \mathbf{R}\right)$ such that

$$
\begin{gathered}
\tilde{H}(u)=H(u) \quad \forall u \in \mathbf{R}^{3},|u| \leq R^{\prime} \\
\sup _{u \in \mathbf{R}^{3}}(1+|u|)|\nabla \tilde{H}(u)|<+\infty \\
\beta_{\tilde{H}}<\beta_{0},
\end{gathered}
$$

(we may take $\tilde{H} \equiv H_{0}$ for $|u| \geq R^{\prime}+1$ )

The two solutions $\underline{u}, \bar{u}$ corresponding to $\tilde{H}$ being such

$$
\|\underline{u}\|_{L^{\infty}},\|\bar{u}\|_{L^{\infty}} \leq R^{\prime}
$$

they are as well solutions to $(I I I)$ for the initial curvature function $H$, and the proof of Theorem 4.2 is complete.

Remark. In fact, Theorem 4.8 assumes that $H$ is smooth. Anyway, let $H$ be only $C^{1}$ : we consider a sequence $H^{n}$ of smooth curvature functions such that $H^{n} \rightarrow H$ in $C^{1}$. If $\left(u^{n}\right)$ is a sequence of solutions to $(I I I)$ corresponding to $H^{n}$, we have

$$
\Delta u^{n}=2 H\left(u^{n}\right) u_{x}^{n} \wedge u_{y}^{n}+f^{n}
$$

with

$$
f^{n}=2\left(H^{n}\left(u^{n}\right)-H\left(u^{n}\right)\right) u_{x}^{n} \wedge u_{y}^{n}
$$

If $H^{n} \rightarrow H$ in $C^{1}$ and $\left(u^{n}\right)$ is bounded in $H^{1} \cap L^{\infty}, f^{n} \rightarrow 0$ in $H^{-1}$. Then, one can apply Theorem 4.6 to the sequences $\left(\underline{u}^{n}\right)$ and $\left(\bar{u}^{n}\right)$, and energy arguments -see for instance (4.22)- impose a strong convergence in $H^{1}$ to solutions $\underline{u}$ and $\bar{u}$ for $(I I I)$ with curvature function $H$. 


\subsection{A priori bounds for smooth solutions.}

Before ending this section, we give the proof of Theorem 4.8. This result is closely related to the conformal invariance of the equation. More precisely, we have (see for instance [36], and references therein) :

Theorem 4.9.- Let $u$ be a smooth solution to (III). The function

$$
\phi(z)=\left|u_{x}\right|^{2}-\left|u_{y}\right|^{2}+2 i u_{x} \cdot u_{y} \quad z=x+i y
$$

is holomorphic in $D^{2}$, i.e.

$$
\frac{\partial \phi}{\partial \bar{z}}=0 \quad \text { in } D^{2}
$$

The function $\phi$ is called the Hopf differential. A simple proof of Theorem 4.9 can be derived multiplying the equation

$$
\Delta u=2 H(u) u_{x} \wedge u_{y}
$$

by $\eta u_{x}+\xi u_{y}$, where $\eta, \xi$ are any test functions in $C_{0}^{\infty}(\Omega)$. The resulting equality yields directly (4.26), in the weak sense.

Note that if $\phi$ is identically equal zero, $u$ is conformal, i.e.

$$
\left|u_{x}\right|^{2}-\left|u_{y}\right|^{2}=u_{x} \cdot u_{y}=0 \text {. }
$$

In other words, the Hopf differential $\phi$ characterizes the "defect" of conformality of the map $u$ : this intuitive statement will be made precise in the course of the proof of Theorem 4.8. Conformal maps have very interesting properties. For instance if $\omega$ is a conformal map in $H^{1}\left(\mathbf{R}^{2}, \mathbf{R}^{2}\right), \omega$ is smooth as standard complex analysis shows (this results extends partially to "quasi-conformal" maps, which belong to $W_{\text {loc }}^{1, p}\left(\mathbf{R}^{2}, \mathbf{R}^{2}\right)$ for some $p>2$, see [11], [12]). If $\omega$ takes its values in $\mathbf{R}^{3}$, this is nolonger true, as easy counterexamples show (one may consider conformal representations of maps with peaks). Nevertheless, if $\omega$ is moreover solution to a nonlinear PDE, we may obtain again uniform bounds, as the following theorem shows.

Theorem 4.10. - Let $\Omega$ be a bounded domain in $\mathbf{R}^{2}, \Lambda \geq 0$, and $\omega$ be a conformal map in $C^{2}\left(\Omega, \mathbf{R}^{k}\right), k \in \mathbf{N}^{*}$, such that

$$
|\Delta \omega| \leq \Lambda|\nabla \omega|^{2}
$$

Then, we have

$$
\|\omega\|_{L^{\infty}} \leq\|\gamma\|_{L^{\infty}}+C\left(\Lambda \int_{D^{2}}|\nabla \omega|^{2}+\left(\int_{D^{2}}|\nabla \omega|^{2}\right)^{1 / 2}\right)
$$


The argument of the proof of this theorem is due to Grüter [13]. We postpone it, and come to the proof of Theorem 4.8

Proof of Theorem 4.8 .

We first remark that $u$ satisfies condition (4.28) of theorem 4.10, with $\Lambda=$ $\|H\|_{L^{\infty}}$. Therefore, if $u$ were conformal, (4.29) would directly yield (4.23), and Theorem 4.8 would be proved. When $u$ is not conformal, a construction is necessary, in order to use again Theorem 4.10. Following an argument of Schoen [31], later extended by Grüter [14], we are going to choose two functions $v_{1}$ and $v_{2}$ such that

$$
\begin{aligned}
\Delta v_{1} & =\Delta v_{2}=0 \\
\int_{D^{2}(1 / 2)} v_{1} & =\int_{D^{2}(1 / 2)} v_{2}=0
\end{aligned}
$$

and

$$
\tilde{\phi}(z)=\left|U_{x}\right|^{2}-\left|U_{y}\right|^{2}+2 i U_{x} \cdot U_{y} \equiv 0
$$

where we have set

$$
\bar{U}=\left(u, v_{1}, v_{2}\right) \in \mathbb{R}^{3} \times \mathbf{R} \times \mathbf{R} .
$$

(4.32) means that $\tilde{\phi}$ is conformal. We readily follow the construction of [14]. For $z_{0} \in D^{2}$ to be determined later, we consider the holomorphic function $\psi$ defined by

$$
\psi(z)=-\frac{1}{4} \int_{z_{0}}^{z} \phi(z) d z
$$

Clearly

$$
\frac{\partial \psi}{\partial z}=-\frac{1}{4} \phi
$$

Take now

$$
v(z)=v_{1}+i v_{2}=\bar{z}+\alpha+\psi(z)
$$

where $\alpha \in \mathbf{C}$ will be chosen later. We have

$$
\frac{\partial}{\partial z} v \cdot \frac{\partial}{\partial z} \bar{v}=\frac{\partial}{\partial z} v \cdot\left(\overline{\frac{\partial}{\partial \bar{z}}} v\right)=-\frac{1}{4} \phi .
$$

On the other hand

$$
\frac{\partial}{\partial z} v \cdot \frac{\partial}{\partial \bar{z}} v=\frac{1}{4}\left(\left|v_{x}\right|^{2}-\left|v_{y}\right|^{2}-2 i v_{x} \cdot v_{y}\right) .
$$

Therefore, (4.32) is satisfied. (4.30) also holds, since

$$
\frac{\partial}{\partial \bar{z}} \cdot \frac{\partial}{\partial z} v=\Delta v=0
$$


We then determine $\alpha$ so that (4.31) is satisfied. Note that

$$
|\alpha| \leq C\left(\int_{D^{2}}|\psi|+1\right)
$$

Combining (4.27) and (4.30), we see that $U$ satisfies (4.28), with $\Lambda=\|H\|_{L^{\infty}}$. Since $U$ is also conformal, we may apply Theorem 4.10. In order to get the estimate (4.23), we are going to distinguish two cases :

(a) $x \in D^{2}(1 / 4)$ (Interior estimates)

(b) $x \in D^{2} \backslash D^{2}(1 / 4)$ (Boundary estimates)

Case(a)- We claim that we may choose $z_{0}$ in (4.33) such that

$$
\int_{D^{2}(1 / 2)}|\psi| \leq C\left(\int_{D^{2}}|\nabla u|^{2}\right),
$$

where $C$ is an absolute constant. Indeed, for $a \in\left(-\frac{1}{2}, \frac{1}{2}\right)$, consider the segment $P(a)=A y \cap D^{2}$, where $A$ is the point $(a, 0)$, and $A_{y}$ denote the line parallel to the $y$-coordinate axis containing $A$. Fubini's theorem ensures that we can choose $a_{0}$ such that

$$
\int_{P\left(a_{0}\right)}|\phi| \leq \int_{D^{2}}|\phi|
$$

We take $z_{0}=a_{0}$. For $z \in D^{2}\left(\frac{1}{2}\right)$, we write

$$
\psi(z)=-\frac{1}{4} \int_{a_{0}}^{a_{0}+i y} \phi(z) d z
$$

Thus

$$
\begin{aligned}
|\psi(z)| & \leq \frac{1}{4} \int_{P\left(a_{0}\right)}|\phi|+\frac{1}{4} \int_{a_{0}+i y}^{x+i y}|\phi| \\
\leq & \frac{1}{4} \int_{D^{2}}|\phi|+\frac{1}{4} \int_{a_{0}+i y}^{x+i y}|\phi|
\end{aligned}
$$

Integrating on $D^{2}\left(\frac{1}{2}\right)$, we obtain (integrating first with respect to $y$ )

$$
\int_{D^{2}(1 / 2)}|\psi| \leq C \int_{D^{2}}|\phi|
$$

On the other hand, we have

$$
|\phi| \leq 2|\nabla u|^{2} \quad \text { on } \quad D^{2}
$$


which, combined with (4.37), yields the claim (4.35).

From the definition of $v$ and (4.34) (4.35) we deduce that

$$
\int_{D^{2}(1 / 2)}|v| \leq C\left(1+\int_{D^{2}}|\nabla u|^{2}\right)
$$

Since $v_{1}$ and $v_{2}$ are harmonic functions satisfying (4.31), we deduce from standard elliptic estimates that

$$
\left\|v_{i}\right\|_{L^{\infty}\left(D^{2}(1 / 3)\right)} \leq C \int_{D^{2}(1 / 2)}|v| \leq C\left(1+\int_{D^{2}}|\nabla u|^{2}\right)
$$

$$
\left\|\nabla v_{i}\right\|_{L^{2}\left(D^{2}(1 / 3)\right)} \leq C \int_{D^{2}(1 / 2)}|v| \leq C\left(1+\int_{D^{2}}|\nabla u|^{2}\right)
$$

Fubini's theorem ensures the existence of $\left.r_{0} \in\right] 1 / 4,1 / 3[$ such that

$$
\int_{\partial D^{2}\left(r_{0}\right)}|\nabla u|^{2} \leq 12 \int_{D^{2}}|\nabla u|^{2}
$$

which implies, by standard arguments, that

$$
\|u\|_{L^{\infty}\left(\partial D^{2}\left(r_{0}\right)\right)} \leq C\|\nabla u\|_{L^{2}\left(D^{2}\right)}+\|\gamma\|_{L^{\infty}\left(\partial D^{2}\right)}
$$

Hence, combining (4.38) and (4.40), the estimate

$$
\|U\|_{L^{\infty}\left(\partial D^{2}\left(r_{0}\right)\right)} \leq C\left(1+\int_{D^{2}}|\nabla u|^{2}\right)+\|\gamma\|_{L^{\infty}\left(\partial D^{2}\right)}
$$

Likewise, (4.39) yields

$$
\|\nabla U\|_{L^{2}\left(D^{2}\left(r_{0}\right)\right)} \leq C\left(1+\int_{D^{2}}|\nabla u|^{2}\right)
$$

Applying Theorem 4.10 to $U$ on $D^{2}\left(r_{0}\right)$, we obtain

$$
\|U\|_{L^{\infty}\left(D^{2}\left(r_{0}\right)\right)} \leq\|U\|_{L^{\infty}\left(\partial D^{2}\left(r_{0}\right)\right)}+C\left(\|H\|_{L^{\infty}} \int_{D^{2}\left(r_{0}\right)}|\nabla U|^{2}+\left(\int_{D^{2}\left(r_{0}\right)}|\nabla U|^{2}\right)^{1 / 2}\right)
$$

which, taking account of (4.41) and (4.42), yields

$$
|u(z)| \leq\|\gamma\|_{L^{\infty}}+C\left(\|H\|_{L^{\infty}}+1\right)\left(1+\int_{D^{2}}|\nabla u|^{2}\right)
$$

for any $z \in D^{2}\left(\frac{1}{4}\right) \cap D^{2}\left(r_{0}\right)$. 
Case $(b)$ - This case reduces to the previous one using the conformal invariance of the equation. Indeed, let $z_{*} \in D^{2} \backslash D^{2}(1 / 4)$. There is a conformal transformation $z \mapsto f(z)$ of the disc $D^{2}$ such that

$$
f\left(D^{2}\right)=D^{2} ; f\left(\partial D^{2}\right)=\partial D^{2} ; f\left(z_{*}\right)=0 .
$$

Since the equation is invariant under conformal transformations, $v=u \circ f$ is also solution to (4.27). Therefore, applying (4.43) for $v$, we get

$$
\begin{aligned}
|v(0)|=\left|u\left(z_{*}\right)\right| & \leq\|v\|_{L^{\infty}\left(\partial D^{2}\right)}+C\left(\|H\|_{L^{\infty}}+1\right)\left(\int_{D^{2}}|\nabla v|^{2}+1\right) \\
& \leq\|\gamma\|_{L^{\infty}}+C\left(\|H\|_{L^{\infty}}+1\right)\left(\int_{D^{2}}|\nabla u|^{2}+1\right) .
\end{aligned}
$$

Thus, (4.43) holds for any $z \in D^{2}$, and the proof of Theorem 4.8 is complete.

We turn now to the proof of Theorem 4.10.

Proof of Theorem 4.10. Let $z_{0}$ be a point in $\Omega$, and $r>0$ such that

$$
\operatorname{dist}\left(\omega\left(z_{0}\right), \omega(\partial \Omega)\right)>r .
$$

We consider the ball $B^{k}\left(\omega\left(z_{0}\right), r\right)$ in the target space $\mathbf{R}^{k}$. Note that

$$
B^{k}\left(\omega\left(z_{0}\right), r\right) \cap \omega(\partial \Omega)=\emptyset .
$$

We define the sets

$$
\begin{aligned}
W\left(z_{0}, r\right) & =\omega^{-1}\left(B^{k}\left(\omega\left(z_{0}\right), r\right)\right) \\
V\left(z_{0}, r\right) & =\partial W\left(z_{0}, r\right)
\end{aligned}
$$

and the function

$$
\Phi\left(z_{0}, r\right)=\int_{W\left(z_{0}, r\right)}|\nabla \omega|^{2}
$$

We simply note $W(r), V(r)$ and $\Phi(r)$, when no confusion is possible.

By Sard's theorem, for almost every $r, V(r)$ is a union of a finite number of smooth curves in $D^{2}$. Let $\nu$ be the outward normal to $V(r)$, and $\tau$ the unit tangent vector, so that $(\nu, \tau)$ is direct. Since $\omega$ is conformal, we have

$$
\left|\frac{\partial \omega}{\partial \nu}\right|=\left|\frac{\partial \omega}{\partial \tau}\right|
$$

and

$$
|\nabla \omega|^{2}=2\left|\frac{\partial \omega}{\partial \nu}\right|^{2}
$$

We set

$$
\Psi(r)=\int_{V(r)}=\left|\frac{\partial|\omega|}{\partial \nu}\right|
$$

Clearly, $\Phi$ is an increasing function of $r$. Following [13], we have : 
Proposition 4.11. - We have

$$
\frac{d}{d r} \Phi(r) \geq 2 \Psi(r)
$$

Proof. Without loss of generality, we may assume that $\omega\left(z_{0}\right)=0$. From (4.44), it follows that

$$
|\nabla \omega|^{2}=2\left|\frac{\partial \omega}{\partial \nu}\right|^{2}=2|\omega|^{2}\left|\frac{\partial}{\partial \nu}\left(\frac{\omega}{|\omega|}\right)\right|^{2}+2\left(\frac{\partial}{\partial \nu}|\omega|\right)^{2}
$$

Therefore

$$
\frac{d}{d r} \Phi(r) \geq 2 \frac{d}{d r}\left(\left.\int_{W(r)}\left|\frac{\partial}{\partial \nu}\right| \omega\right|^{2}\right)=2 \frac{d}{d r}\left(\int_{W(r)}|\nabla| \omega||^{2}\right)
$$

since $\left|\frac{\partial}{\partial \nu}\right| \omega||=|\nabla| \omega||$. Indeed, $V(r)$ being a level set for $|\omega|, \frac{\partial}{\partial \tau}|\omega|=0$. We deduce from the coarea formula of Federer that

$$
\int_{W(r)}|\nabla| \omega||^{2}=\int_{0}^{r}\left(\int_{V(\sigma)}\left|\frac{\partial|\omega|}{\partial \nu}\right|\right) d \sigma
$$

Thus

$$
\frac{d}{d r} \int_{W(r)}|\nabla| \omega||^{2}=\int_{V(r)}\left|\frac{\partial|\omega|}{\partial \nu}\right|=\Psi(r)
$$

(4.45) (4.46) give the announced inequality.

We have then the following result :

Proposition 4.12. - We have the inequality

$$
2 \Phi(r)-r \frac{d}{d r} \Phi(r) \leq 2 \Lambda r \Phi(r) .
$$

Proof. Integrating by parts, we have

$$
\begin{aligned}
\Phi(r)=\int_{W(r)}|\nabla \omega|^{2} & =\int_{W(r)}-\Delta \omega \cdot \omega+\int_{V(r)} \omega \cdot \frac{\partial \omega}{\partial \nu} \\
& \leq \Lambda \int_{W(r)}|\omega||\nabla \omega|^{2}+r \int_{V(r)}\left|\frac{\partial|\omega|}{\partial \nu}\right| \\
& \leq \Lambda \int_{W(r)}|\omega||\nabla \omega|^{2}+r \Psi(r) \\
& \leq \Lambda r \Phi(r)+\frac{1}{2} r \frac{d}{d r} \Phi(r),
\end{aligned}
$$

by Proposition 4.11. This yields the inequality.

Next, we prove : 
Proposition 4.13.- Assume that $\left|\nabla \omega\left(x_{0}\right)\right| \neq 0$. Then

$$
\overline{\lim }_{r \rightarrow 0} \frac{\Phi(r)}{r^{2}} \geq 2 \pi
$$

Proof. Since $\omega$ is $C^{2}$, there exist $C$ constant such that for $z$ close to $z_{0}$

$$
\left|\nabla \omega(z)-\nabla \omega\left(z_{0}\right)\right| \leq C\left|z-z_{0}\right|
$$

On the other hand, the conformality of $\omega$ yields, for any vector $n$ in $\mathbb{R}^{2}$

$$
|n . \nabla \omega(z)|=\frac{1}{\sqrt{2}}\left|\nabla \omega\left(z_{0}\right)\right| .
$$

Therefore

$$
\left|\omega(z)-\omega\left(z_{0}\right)\right| \leq \frac{1}{\sqrt{2}}\left|\nabla \omega\left(z_{0}\right)\right|\left|z-z_{0}\right|+\frac{C}{\sqrt{2}}\left|z-z_{0}\right|^{2} .
$$

It follows that

$$
D^{2}\left(z_{0}, \rho\right) \subset W(r)
$$

with $\rho>0$ such that

$$
\frac{1}{\sqrt{2}}\left|\nabla \omega\left(z_{0}\right)\right| \rho+\frac{C}{\sqrt{2}} \rho^{2}=r
$$

Then, the inequality

$$
\begin{aligned}
\Phi(r) \geq \int_{D^{2}\left(z_{0}, \rho\right)}|\nabla \omega|^{2} & \geq \int_{D^{2}\left(z_{0}, \rho\right)}\left(\left|\nabla \omega\left(z_{0}\right)\right|-C\left|z-z_{0}\right|\right)^{2} \\
& \geq \pi\left(\left|\nabla \omega\left(z_{0}\right)\right|^{2} \rho^{2}+\frac{C^{2}}{2} \rho^{4}-\frac{4 C}{3}\left|\nabla \omega\left(z_{0}\right)\right| \rho^{3}\right)
\end{aligned}
$$

yields

$$
\frac{\Phi(r)}{r^{2}} \geq 2 \pi-C^{\prime} r
$$

where $C^{\prime}$ is some constant, as $r \rightarrow 0$. The proposition follows.

The last result that we need is the following.

Proposition 4.14.- For any $r, 0<r \leq \frac{1}{2 \Lambda}$,

$$
\Phi(r) \geq \frac{2 \pi}{e} r^{2} .
$$

Proof. We compute

$$
\frac{d}{d r}\left(\frac{\Phi(r)}{r^{2}}\right)=\frac{1}{r^{2}} \frac{d}{d r} \Phi(r)-\frac{2}{r^{3}} \Phi(r)=\frac{1}{r^{3}}\left(r \frac{d}{d r} \Phi(r)-2 \Phi(r)\right)
$$


From Proposition 4.12, we deduce that

$$
-\frac{d}{d r}\left(\frac{\Phi(r)}{r^{2}}\right) \leq 2 \Lambda \frac{\Phi(r)}{r^{2}}
$$

Setting

$$
\chi(r)=\frac{\Phi(r)}{r^{2}}
$$

(4.47) writes as

$$
\frac{d}{d r}(\ln \chi(r)) \geq-2 \Lambda
$$

For $\varepsilon \in(0, r)$, we integrate (4.48) between $\varepsilon$ and $r$. We are led to

$$
\ln \chi(r) \geq-2 \Lambda r+\ln \chi(\varepsilon) .
$$

We let $\varepsilon$ go to zero. In view of Proposition 4.13, we obtain

$$
\ln \chi(r) \geq-2 \Lambda r+\ln 2 \pi
$$

that is

$$
\chi(r) \geq 2 \pi e^{-2 \Lambda r}
$$

This yields the conclusion.

Proof of Theorem 4.10 completed. Let $z_{1}$ be a point in $D^{2}$. For $\ell=1, \cdots, k$, we denote by $\omega^{\ell}(z)$ the $\ell$-th coordinate of $\omega$. $\omega$ being continuous, we have

$$
\omega^{\ell}\left(\partial D^{2}\right)=[\alpha, \beta] \quad \alpha, \beta \in \mathbb{R} .
$$

If $\omega^{\ell}\left(z_{1}\right) \in[\alpha, \beta]$, there is nothing to prove. Otherwise, $\omega^{\ell}\left(z_{1}\right)<\alpha$ or $\omega^{\ell}\left(z_{1}\right)>\beta$. We consider first the case

$$
\omega^{\ell}\left(z_{1}\right)>\beta
$$

We set

$$
\begin{aligned}
\bar{\Lambda} & =\max \left(\Lambda,\left(\int_{D^{2}}|\nabla \omega|^{2}\right)^{-1 / 2}\right) \\
d & =\omega^{\ell}\left(z_{1}\right)-\beta .
\end{aligned}
$$

Let $p$ such that

$$
d \bar{\Lambda}-1 \leq p<d \bar{\Lambda}
$$

We claim that

$$
p \leq \frac{2 e}{\Pi} \bar{\Lambda}^{2} \int_{D^{2}}|\nabla \omega|^{2} .
$$


Indeed, if $p \neq 0$, since $\omega$ is continuous, we may find $(p-1)$ points $z_{2}, \cdots, z_{p}$ in $D^{2}$ such that, for any $i, j \in\{1, \cdots, p\}, i \neq j$

$$
\left|\omega^{\ell}\left(z_{i}\right)-\omega^{\ell}\left(z_{j}\right)\right| \geq \frac{1}{\bar{\Lambda}}
$$

and, for any $i \in\{1, \cdots, p\}$

$$
\omega^{\ell}\left(z_{i}\right) \geq \beta+\frac{1}{\bar{\Lambda}}
$$

We deduce from (4.50) that

$$
B^{k}\left(\omega\left(z_{i}\right), \frac{1}{2 \bar{\Lambda}}\right) \cap B^{k}\left(\omega\left(z_{j}\right), \frac{1}{2 \bar{\Lambda}}\right)=\emptyset \quad \forall i, j, i \neq j,
$$

and from (4.51) that

$$
B^{k}\left(\omega\left(z_{i}\right), \frac{1}{2 \bar{\Lambda}}\right) \cap \omega\left(\partial D^{2}\right)=\emptyset \quad \forall i
$$

It follows that

$$
\begin{aligned}
\int_{D^{2}}|\nabla \omega|^{2} & \geq \int_{\cup_{i=1}^{p} \omega^{-1}\left(B^{k}\left(\omega\left(z_{i}\right), \frac{1}{2 \bar{\Lambda}}\right)\right)}|\nabla \omega|^{2} \\
& \geq \sum_{i=1}^{p} \int_{W\left(z_{i}, \frac{1}{2 \bar{\Lambda}}\right)}|\nabla \omega|^{2} \\
& \geq \sum_{i=1}^{p} \Phi\left(z_{i}, \frac{1}{2 \bar{\Lambda}}\right) .
\end{aligned}
$$

Applying Proposition 4.14, we know that

$$
\Phi\left(z_{i}, \frac{1}{2 \bar{\Lambda}}\right) \geq \frac{2 \pi}{e} \frac{1}{4 \bar{\Lambda}^{2}} .
$$

Thus

$$
\int_{D^{2}}|\nabla \omega|^{2} \geq\left(\frac{\pi}{2 e} \cdot \frac{1}{\bar{\Lambda}^{2}}\right) p
$$

and (4.49) follows.

As a consequence, we obtain

$$
d \leq \frac{p+1}{\bar{\Lambda}} \leq \frac{1}{\bar{\Lambda}}\left(1+\frac{2 e}{\pi} \bar{\Lambda}^{2} \int_{D^{2}}|\nabla \omega|^{2}\right) .
$$

From the definition of $\bar{\Lambda}$, we have

$$
\bar{\Lambda}^{2} \int_{D^{2}}|\nabla \omega|^{2} \geq 1
$$


whence

$$
\begin{aligned}
\omega^{\ell}\left(z_{1}\right)-\beta=d & \leq\left(1+\frac{2 e}{\pi}\right) \bar{\Lambda} \int_{D^{2}}|\nabla \omega|^{2} \\
& \leq C\left(\Lambda \int_{D^{2}}|\nabla \omega|^{2}+\left(\int_{D^{2}}|\nabla \omega|^{2}\right)^{1 / 2}\right) .
\end{aligned}
$$

Likewise, if $\omega^{\ell}\left(z_{1}\right)<\alpha$, we obtain

$$
\alpha-\omega^{\ell}\left(z_{1}\right) \leq C\left(\Lambda \int_{D^{2}}|\nabla \omega|^{2}+\left(\int_{D^{2}}|\nabla \omega|^{2}\right)^{1 / 2}\right)
$$

and the proof of Theorem 4.10 is complete.

\section{The Plateau Problem}

In this section, we deal with solutions to the original Plateau problem, that is with maps $u: D^{2} \rightarrow \mathbf{R}^{3}$ which satisfy

$$
\Delta u=2 H(u) u_{x} \wedge u_{y} \text { in } D^{2}
$$

$$
\left|u_{x}\right|^{2}-\left|u_{y}\right|^{2}=u_{x} \cdot u_{y}=0 \text { in } D^{2}
$$

$\left.u\right|_{\partial D^{2}}$ is a continuons monotone parametrization of $\Gamma$.

Similarly to Theorem 4.2 , we have :

Theorem 5.1. - Assume that $H_{0} \in R-\{0\}$ is such that $\left|H_{0}\right| .|| \Gamma \|_{L^{\infty}}<1$ with $\Gamma$ not reduced to a point.

There exists a number $\alpha \geq 0$ such that for any curvature function $H \in C^{1}\left(\mathbf{R}^{3}, \mathbf{R}\right)$ which satisfies

$$
\left\|H-H_{0}\right\|_{L^{\infty}\left(\mathbf{R}^{3}, \mathbf{R}\right)} \leq \alpha
$$

(P) admits at least two solutions.

Proof. The existence of a first solution to (P) is well known. It suffices to minimize $J_{H}(\underline{u})$, where $\underline{u}$ is given by Theorem 2.1 , with respect to the parametrization $\gamma$ of $\Gamma$. The infimum is achieved for some $\underline{u}_{p}$ which is a "small" solution to $(\mathrm{P})$ - see Hildebrandt [17].

One could think to use the same kind of procedure to obtain a second solution : minimize $J_{H}(\bar{u})$, where $\bar{u}$ is given by Theorem 4.2 , with respect to the parametrization $\gamma$ of $\Gamma$. This was realized by Brezis and Coron [7], in the case of constant H. However, 
the extension of this method to variable $H$ would require some assumptions on the derivatives of $H$. To avoid this, one may also proceed as in the previous section, with a set of variations including the parametrizations of the boundary. The solution of the equation obtained by this method is also stationary with respect to the variations of $\gamma$ parametrization of $\Gamma$, and then is conformal - see [36-Proposition II.2.8].

The proof of Theorem 5.1 follows the argument developped by Struwe in the case of constant $H$ [36,Section IV] ; we only sketch it.

Let $\gamma$ be any fixed, smooth and monotone parametrization of $\Gamma$. Instead of $H_{\gamma}^{1}$, the set of variations is now

$$
E=B \times H_{0}^{1}\left(D^{2}, \mathbf{R}^{3}\right)
$$

with

$$
\begin{gathered}
B=\left\{v \in C^{0}(\mathbf{R}, \mathbf{R}) / v \text { monotone } ; v(t+2 \pi)=v(t)+2 \pi, \forall t ;\right. \\
\left.v\left(\frac{2 \pi k}{3}\right)=\frac{2 \pi k}{3}, \forall k \in \mathbf{Z} ; \int_{D^{2}}|\nabla P v|^{2}<+\infty\right\}
\end{gathered}
$$

where $P v$ denotes the harmonic extension of $\gamma \circ v$ to $D^{2}$, i.e.

$$
\left\{\begin{array}{l}
\Delta P v=0 \text { in } D^{2} \\
P v=\gamma \circ v \text { on } \partial D^{2}
\end{array}\right.
$$

The three-points condition is introduced in view of the invariance of the problem with respect to the conformal transformations of the disc. Note that $E$ may be embedded in

$$
F=\left(\{i d\}+H^{1 / 2} \cap C^{0}(\mathbf{R} / 2 \pi)\right) \times H_{0}^{1}\left(D^{2}, \mathbf{R}^{3}\right)
$$

and equipped with the induced topology.

For $U=\left(u_{1}, u_{2}\right) \in F$, we define the functional.

$$
\begin{gathered}
\mathcal{J}_{H}(U)=J_{H}(u) \\
u=P u_{1}+u_{2}
\end{gathered}
$$

$\mathcal{J}_{H}$ is not $C^{1}$ with respect to the second variable $u_{2}$, but admits a continuons derivative in any direction of $H_{0}^{1} \cap L^{\infty}$. Thus we may define

$$
\left\|\mathcal{J}_{H}^{\prime}(U)\right\|=\sup _{\substack{V \in E \\ u_{2}-v_{2} \in H_{0}^{1} \cap L^{\infty} \\\|U-V\|_{F}=1}}\left|\mathcal{J}_{H}^{\prime}(U) . U-V\right| \in \overline{\mathbf{R}}_{+}
$$

We have :

Lemma 5.2.- $U \in E$ is critical for $\mathcal{J}_{H}$, i.e $\left\|\mathcal{J}_{H}^{\prime}(U)\right\|=0$ if and only if $U$ is solution to $(P)$. 
This lemma follows from [36, Lemma III 4.4].

Following the strategy of the previous section, we apply the mountain-pass lemma to the functional $\mathcal{J}_{H}$, starting from the small solution $\underline{U}=\left(\left.\gamma^{-1} \circ \underline{u}_{p}\right|_{\partial D^{2}}, \underline{u}_{p}\right) ; \underline{U}$ is by definition a local minimum for $\mathcal{J}_{H}$. The existence of $r, \rho>0$ such that

$$
\mathcal{J}_{H}(U)>\rho \text { for any } U \in E,\|U-V\|_{F}=r
$$

follows from [35, Section 4], provided that $\beta_{H}$ is small enough. The existence of $U_{0} \in E,\left\|U_{0}\right\|_{F}>r, \mathcal{J}_{H}\left(U_{0}\right)<\mathcal{J}_{H}(\underline{U})$ is straightforward. Setting then

$$
d_{H}=\inf _{p \in P} \sup _{t \in[0,1]} \mathcal{J}_{H}(p(t)
$$

with

$$
P=\left\{p \in C^{0}([0,1], E) \text { s.t. } p(0)=\underline{U}, p(1)=U_{0}\right\},
$$

there exists a sequence $\left(U^{n}\right)$ of $E$ such that

$$
\begin{aligned}
\mathcal{J}_{H}\left(U^{n}\right) & \rightarrow d_{H} \\
\left\|\mathcal{J}_{H}^{\prime}\left(U^{n}\right)\right\| & \rightarrow 0 .
\end{aligned}
$$

The problem is the following : in order to be able to extract a convergent subsequence of $\left(U^{n}\right)$, we need to know that $\left(U^{n}\right)$ remains bounded in $F$. Unfortunately, the argument of Lemma 4.4 does not allow to conclude, when the boundary values are not fixed. To obtain boundedness, Struwe suggests the following strategy.

Lemma 5.3.- ([36 -Lemma III 4.10])

$$
\text { The map } \xi \in]-1,1\left[\rightarrow \frac{d_{(1+\xi) H}}{1+\xi} \quad\right. \text { is not increasing . }
$$

Proof. For $\xi_{1}<\xi_{2}$, there holds

$$
\frac{1}{1+\xi_{1}} J_{\left(1+\xi_{1}\right) H}(U)-\frac{1}{1+\xi_{2}} J_{\left(1+\xi_{2}\right) H}(U)=\frac{1}{2} \frac{\xi_{2}-\xi_{1}}{\left(1+\xi_{1}\right)\left(1+\xi_{2}\right)} \int_{D^{2}}|\nabla \tilde{u}|^{2} \geq 0
$$

We assume that $\xi_{1}$ and $\xi_{2}$ are sufficiently small, so that we still have

$$
\left(1+\xi_{i}\right)\left|H_{0}\right| \cdot\|\Gamma\|_{L^{\infty}}<1 \quad i=1,2 .
$$

$\left(1+\xi_{i}\right) H$ is close to $\left(1+\xi_{i}\right) H_{0}$, and the mountain-pass lemma applies. Let $p^{n} \in P$ be a sequence of minimizing paths for $\left(1+\xi_{1}\right) H$ i.e.

$$
\sup _{t \in[0,1]} \mathcal{J}_{\left(1+\xi_{1}\right) H} \quad\left(p^{n}(t)\right) \underset{n \rightarrow+\infty}{\rightarrow} d_{\left(1+\xi_{1}\right) H}
$$


and $t^{n} \in[0,1]$ be such that

$$
\mathcal{J}_{\left(1+\xi_{2}\right) H}\left(p^{n}\left(t^{n}\right)\right)=\sup _{t \in[0,1]} \mathcal{J}_{\left(1+\xi_{2}\right) H}\left(p^{n}(t)\right) \geq d_{\left(1+\xi_{2}\right) H}
$$

We have

$$
\begin{aligned}
\frac{1}{1+\xi_{1}} d_{\left(1+\xi_{1}\right) H} & \geq \underline{\lim } \frac{1}{1+\xi_{1}} \mathcal{J}_{\left(1+\xi_{1}\right) H}\left(p^{n}\left(t^{n}\right)\right) \\
& \geq \underline{\lim } \frac{1}{1+\xi_{2}} \mathcal{J}_{\left(1+\xi_{2}\right) H}\left(p^{n}\left(t^{n}\right)\right) \\
& \geq \frac{1}{1+\xi_{2}} d_{\left(1+\xi_{2}\right) H} .
\end{aligned}
$$

Hence the lemma follows.

Therefore, the map $\xi \mapsto \frac{d_{(1+\xi) H}}{1+\xi}$ is almost everywhere differentiable near 0 . Still following [36], we set

$$
\begin{aligned}
& \mathcal{D}=\left\{\xi_{0} \in\right]-1,1\left[/ d_{(1+\xi) H} \text { is defined near } \xi_{0}\right. \text {, and } \\
& \left.\varlimsup_{\xi \rightarrow \xi_{0}} \frac{1}{\xi_{0}-\xi}\left(\frac{d_{(1+\xi) H}}{1+\xi}-\frac{d_{\left(1+\xi_{0}\right) H}}{1+\xi_{0}}\right)<+\infty\right\}
\end{aligned}
$$

As $\mathcal{D}$ is a dense neighbourhood of 0 , we may approximate $H$ by curvature functions $H^{n} \in \mathcal{D}, H^{n}=\left(1+\xi^{n}\right) H, \xi^{n} \rightarrow 0$ as $n \rightarrow+\infty$.

Then, we have the result :

Lemma 5.4.- For $n$ large enough, $\mathcal{J}_{H^{n}}$ has a critical point at level $d_{H^{n}}$.

Proof. For fixed $n$, we choose a sequence $\xi_{k}^{n}$ such that $\xi_{k}^{n}>\xi^{n}$ and $\xi_{k}^{n} \rightarrow \xi^{n}$ as $k \rightarrow+\infty$. Let $\left(p_{k}^{n}\right)$ be a minimizing sequence of paths for $H^{n}$ such that

$$
\sup _{t \in[0,1]} \mathcal{J}_{H^{n}}\left(p_{k}^{n}(t)\right) \leq d_{H^{n}}+\xi_{k}^{n}-\xi^{n}
$$

Let $t \in[0,1]$ be such that

$$
\mathcal{J}_{H_{k}^{n}}\left(p_{k}^{n}(t)\right) \geq d_{H_{k}^{n}}-\xi_{k}^{n}+\xi^{n}
$$

with $H_{k}^{n}=\left(1+\xi_{k}^{n}\right) H$. Setting

$$
p_{k}^{n}(t)=U=\left(u_{1}, u_{2}\right), \quad \text { and } \quad u=P u_{1}+u_{2}
$$


(5.1) yields, as $H^{n} \in \mathcal{D}$

$$
\begin{aligned}
\int_{D^{2}}|\nabla u|^{2} & \leq 2 \frac{\left(1+\xi_{k}^{n}\right)\left(1+\xi^{n}\right)}{\xi_{k}^{n}-\xi^{n}}\left(\frac{d_{H^{n}}+\xi_{k}^{n}-\xi^{n}}{1+\xi^{n}}-\frac{d_{H_{k}^{n}}-\xi_{k}^{n}+\xi^{n}}{1+\xi_{k}^{n}}\right) \\
& \leq 2 \frac{\left(1+\xi_{k}^{n}\right)\left(1+\xi^{n}\right)}{\xi_{k}^{n}-\xi^{n}}\left(\frac{d_{H^{n}}}{1+\xi^{n}}-\frac{d_{H_{k}^{n}}}{1+\xi_{k}^{n}}\right)+4+2 \xi^{n}+2 \xi_{k}^{n} \\
& \leq c_{n}<+\infty
\end{aligned}
$$

for $k$ large enough.

Suppose now that there exits $\eta>0$ such that

$$
\left\|\mathcal{J}_{H_{k}^{n}}^{\prime}(U)\right\| \geq \eta,
$$

for any $k$ large enough, and any $U \in E$ such that

$$
\begin{aligned}
\mathcal{J}_{H_{k}^{n}}(U) & \geq d_{H_{k}^{n}}-\xi_{k}^{n}+\xi^{n} \\
\int_{D^{2}}|\nabla u|^{2} & \leq c_{n} .
\end{aligned}
$$

As in the previous section, one can build a pseudo-gradient vector field for $\mathcal{J}_{H_{k}^{n}}$, which is also a pseudo-gradient vector field for $\mathcal{J}_{H^{n}}$ on this bounded set. Using this vector field, one could deform the paths $p_{k}^{n}$ into paths linking $\underline{U}$ to $U_{0}$ under the level $d_{H_{k}^{n}}$ for $\mathcal{J}_{H_{k}^{n}}$, a contradiction.

We obtain therefore the existence of a sequence $\left(U_{k}^{n}\right)$ of $E$ such that

$$
\begin{gathered}
\int_{D^{2}}\left|\nabla u_{k}^{n}\right|^{2} \leq c_{n} \\
\lim _{k} \mathcal{J}_{H_{k}^{n}}\left(U_{k}^{n}\right)=\lim _{k} \mathcal{J}_{H^{n}}\left(U_{k}^{n}\right)=d_{H^{n}} \\
\lim _{k}\left\|\mathcal{J}_{H_{k}^{n}}^{\prime}\left(U_{k}^{n}\right)\right\|=\lim _{k}\left\|\mathcal{J}_{H^{n}}^{\prime}\left(U_{k}^{n}\right)\right\|=0 .
\end{gathered}
$$

In order to prove that the sequence $\left(U_{k}^{n}\right)$ converges strongly to a critical point of $J_{H^{n}}$, we concentrate our attention to the part $P\left(u_{1}\right)_{k}^{n}$ of $u_{k}^{n}=P\left(u_{1}\right)_{k}^{n}+\left(u_{2}\right)_{k}^{n} \quad\left(U_{k}^{n}=\right.$ $\left.\left(\left(u_{1}\right)_{k}^{n},\left(u_{2}\right)_{k}^{n}\right)\right)$. Note that $P\left(u_{1}\right)_{k}^{n}$ is bounded in $H^{1}$, since

$$
\int_{D^{2}}\left|\nabla u_{k}^{n}\right|^{2}=\int_{D^{2}}\left|\nabla P\left(u_{1}\right)_{k}^{n}\right|^{2}+\int_{D^{2}}\left|\nabla\left(u_{2}\right)_{k}^{n}\right|^{2} \leq c_{n}
$$

and

$$
\left\|P\left(u_{1}\right)_{k}^{n}\right\|_{L^{\infty}}=\|\Gamma\|_{L^{\infty}} .
$$

We need the following property : 


\section{Lemma 5.5.- The injection}

$\left\{w \in H^{1}\left(D^{2}, \mathbf{R}^{3}\right) /\left.w\right|_{\partial D^{2}} \in C^{0}\left(\partial D^{2}, \mathbf{R}^{3}\right) ;\left.w\right|_{\partial D^{2}}\right.$ is a monotone parametrization of $\left.\Gamma ; w\left(e^{\frac{2 i \pi}{3} k}\right)=e^{\frac{2 i \pi}{3} k}, k=0,1,2\right\} \rightarrow C^{0}\left(\partial D^{2}, \mathbf{R}^{3}\right)$

is compact.

The proof of this lemma, which relies on a result of Courant and Lebesgue, is given by Struwe [36, Lemma I.4.3].

Up to a subsequence, we may then assume that

$$
\begin{aligned}
& \left(u_{2}\right)_{k}^{n} \rightarrow\left(u_{2}\right)_{k}^{n} \quad \text { in } \quad H_{0}^{1} \\
& P\left(u_{1}\right)_{k}^{n} \rightarrow P\left(u_{1}\right)^{n} \text { in } H^{1} \\
& P\left(u_{1}\right)_{k}^{n} \rightarrow P\left(u_{1}\right)^{n} \quad \text { uniformly in } D^{2} .
\end{aligned}
$$

It follows that $P\left(u_{1}\right)_{k}^{n} \rightarrow P\left(u_{1}\right)^{n}$ strongly in $H^{1}$ - see [36, Lemmas II.2.10 and IV.4.5].

We can now apply the following result, proved in Appendix :

Theorem 5.6.- Assume that $H: \mathbb{R}^{3} \rightarrow \mathbb{R}$ satisfies

$$
\sup _{u \in \mathbf{R}^{3}}(|H(u)|+(1+\mid u)|\nabla H(u)|) \leq \infty
$$

Let $\left(u^{n}\right)$ be a bounded sequence in $H^{1}\left(D^{2}, \mathbf{R}^{3}\right)$ such that

$$
\Delta u^{n}=2 H\left(u^{n}\right) u_{x}^{n} \wedge u_{y}^{n}+f^{n} \quad \text { in } \quad D^{2}
$$

with

$$
f^{n} \rightarrow 0 \text { in } H^{-1}
$$

Furthermore, we assume that $\left.u^{n}\right|_{\partial D^{2}}$ is bounded in $H^{1 / 2} \cap L^{\infty}\left(\partial D^{2}, \mathbf{R}^{3}\right)$, and converges to some limit $\gamma$, strongly in $H^{1 / 2}$, and uniformly. Then, there exist

(i) $u^{0} \in H_{\gamma}^{1}$, solution to (III)

(ii) a finite number of nonconstant solutions $\omega_{1}, \cdots, \omega_{p}$ of $\Delta \omega=2 H(\omega) \omega_{x} \wedge \omega_{y}$ on whole $\mathbf{R}^{2}$

(iii) sequences $\left(a_{1}^{n}\right), \cdots,\left(a_{p}^{n}\right)$ in $D^{2}$

(iv) sequences $\left(\varepsilon_{1}^{n}\right), \cdots,\left(\varepsilon_{p}^{n}\right)$ in $\mathbf{R}_{+}^{*}, \lim _{n \rightarrow+\infty} \varepsilon_{i}^{n}=0, \forall i$

such that, up to a subsequence, we have

$$
\left\|u^{n}-u^{0}-\sum_{i=1}^{p} \omega_{i}\left(\frac{-a_{i}^{n}}{\varepsilon_{i}^{n}}\right)\right\|_{H^{1}} \underset{u \rightarrow+\infty}{\longrightarrow} 0
$$


and

$$
\begin{gathered}
\int_{D^{2}}\left|\nabla u^{n}\right|^{2}=\int_{D^{2}}\left|\nabla u^{0}\right|^{2}+\sum_{i=1}^{p} \int_{\mathbf{R}^{2}}\left|\nabla \omega^{i}\right|^{2}+o(1), \\
J_{H}\left(u^{n}\right)=J_{H}\left(u^{0}\right)+\sum_{i=1}^{p} J_{H}\left(\omega_{i}\right)+o(1) .
\end{gathered}
$$

We apply the above result to sequence $\left(u_{k}^{n}\right)_{k}$. Similar arguments as in the previous section show that the convergence of $\left(u_{k}^{n}\right)_{k}$ has to be strong. Passing to the limit $k \rightarrow+\infty$, we obtain a sequence $\left(u^{n}\right), u^{n} \in H_{\gamma^{n}}^{1}$, which are the parametrization of surfaces with mean curvature $H^{n}$ spanning $\Gamma$. This completes the proof of Lemma 5.4 .

To complete the proof of Theorem 5.1, it suffices to remark that $\left(u^{n}\right)$ will still provide us with a Palais-Smale sequence for $J_{H}$ which is bounded. Indeed, we have the following lemma.

Lemma 5.7.- Any solution to $(P)$ satisfies the estimate

$$
\int_{D^{2}}|\nabla u|^{2} \leq \frac{1}{\frac{1}{2}-\beta_{H}}\left(3 J_{H}(u)+L(\Gamma)^{2}\right)
$$

where $L(\Gamma)$ denotes the length of $\Gamma$.

Proof. We follow the argument of Struwe [36, Theorem IV 4.2]. Multiplying the equation

$$
\Delta u=2 H(u) u_{x} \wedge u_{y}
$$

by $u$, and integrating on $D^{2}$, we obtain

$$
\begin{aligned}
0 & =\int_{D^{2}}\left(-\Delta u+2 H(u) u_{x} \wedge u_{y}\right) \cdot u \\
& \leq \int_{D^{2}}\left(|\nabla u|^{2}+2 Q(u) u_{x} \wedge u_{y}\right)+\beta_{H} \int_{D^{2}}|\nabla u|^{2}-\int_{\partial D^{2}} \frac{\partial u}{\partial n} \cdot u \\
& \leq 3 J_{H}(u)-\left(\frac{1}{2}-\beta_{H}\right) \int_{D^{2}}|\nabla u|^{2}+\int_{\partial D^{2}}\left|\frac{\partial u}{\partial n}\right|\|u\|_{L^{\infty}} \\
& \leq 3 J_{H}(u)-\left(\frac{1}{2}-\beta_{H}\right) \int_{D^{2}}|\nabla u|^{2}+\|\Gamma\|_{L^{\infty}} L(\Gamma)
\end{aligned}
$$

since $u$ is conformal. With a suitable choice of the origin, $\|\Gamma\|_{L^{\infty}} \leq L(\Gamma)$, and the results follows.

The same arguments as before show now that $\left(u_{n}\right)$ converges strongly in $H^{1}$ to a second solution to $(P)$. As in the previous section, the estimate of Theorem 4.8 allows to replace the condition of finiteness and smallness of $\beta_{H}$ by the only assumption of the closeness of $H$ to $H_{0}$ in $L^{\infty}$-norm, and Theorem 5.1 is proved. 


\section{Additional geometrical remarks}

\subsection{Imbedded spheres with prescribed mean curvature.}

As a consequence of our analysis, we are led to the following.

Theorem 6.1.- There exists some absolute constant $\alpha>0$, such that if

$$
H_{0} \in \mathbf{R}-\{0\} ; H \in C^{1}\left(\mathbf{R}^{3}, \mathbf{R}\right)
$$

satisfy

$$
\frac{1}{\left|H_{0}\right|}\left\|H-H_{0}\right\|_{L^{\infty}}<\alpha
$$

there exists some map $u: S^{2}\left(\simeq \mathbf{R}^{2}\right) \rightarrow \mathbb{R}^{3}, u \not \equiv$ constant, such that

$$
\begin{gathered}
\Delta u=2 H(u) u_{x} \wedge u_{y} \\
\left|u_{x}\right|^{2}-\left|u_{y}\right|^{2}=u_{x} \cdot u_{y}=0,
\end{gathered}
$$

that is, $u$ is a conformal parametrization of an immersed sphere in $\mathbb{R}^{3}$, whose mean curvature at the point $u(x, y)$ is $H(u(x, y))$.

Sketch of the proof. Set $\tilde{H}(u)=\frac{1}{H_{0}} H\left(\frac{u}{H_{0}}\right)$. It follows from (6.1) that

$$
\|\tilde{H}-1\|_{L^{\infty}}<\alpha
$$

Let $\gamma: \partial D^{2} \rightarrow \mathbf{R}^{3}$ be a smooth map, and for $n \in \mathbf{N}^{*}$ we define

$$
\gamma^{n}=\frac{1}{n} \gamma
$$

Theorem 4.2 provides us with a large solution $u^{n}$ to $(I I I)$, for $\tilde{H}$ as curvature function and $\gamma^{n}$ as boundary values, as soon as $n$ is large enough.

The constant $\alpha$ in Theorem 4.2. may depend on the boundary values $\gamma$. However, one can choose $\alpha$ independently of $\gamma$ as $\|\gamma\|_{L^{\infty}}$ goes to zero, as it is easily seen returning to the arguments of the proof.

Let $h: D^{2} \rightarrow \mathbf{R}^{3}$ be the harmonic extension of $\gamma$, and $h^{n}=\frac{1}{n} h$. We set

$$
v^{n}=u^{n}-h^{n}
$$


Clearly, $\left(v^{n}\right)$ is a Palais-Smale sequence for $J_{\tilde{H}}$, with $v^{n}=0$ on $\partial D^{2}$. Theorem 4.6 then applies, i.e., up to a subsequence

$$
\left\|v^{n}-v^{0}-\sum_{i=1}^{p} \omega_{i}\left(\cdot-a_{i}^{n}\right)\right\|_{H^{1}} \rightarrow 0
$$

$$
J_{\tilde{H}}\left(v^{n}\right)=J_{\tilde{H}}\left(v^{0}\right)+\sum_{i=1}^{p} J_{\tilde{H}}\left(\omega^{i}\right)+o(1),
$$

$v^{0}$ satisfies

$$
\Delta v^{0}=2 \tilde{H}\left(v^{0}\right) v_{x}^{0} \wedge v_{y}^{0} \quad \text { in } \quad D^{2} ; v^{0}=0 \quad \text { on } \quad \partial D^{2} .
$$

Therefore, $v^{0} \equiv 0$, by a result of Wente [42].

On the other hand, $u^{n}$ being a "large" solution to $(I I I),(6.5)$ implies that $p=1$. Hence the existence of $\omega$ satisfying (6.2) in $\mathbf{R}^{2}$, with $\tilde{H}$ as curvature function, $\omega \not \equiv$ constant. Thus

$$
u=\frac{\omega}{H_{0}}
$$

satisfies (6.2). Moreover, the boundedness of $\int_{\mathbf{R}^{2}}|\nabla u|^{2}$ ensures that $u$ is conformal see [42], or the proof of Lemma 4.7. This completes the proof of Theorem 6.1.

Remark. 1 - One might prove that these immersed spheres are actually embedded, condition (6.1) ensuring that these spheres are close, in a suitable sense, of round spheres of radius $\frac{1}{\left|H_{0}\right|}$

2 - In fact, Theorem 6.1 provides us with infinitely many non trivial solutions to (6.2) (6.3). Indeed, instead of concentrating the boundary values of the large solutions to $(I I I)$ at zero, one could by translation concentrate them at any given point in $\mathbf{R}^{2}$. Therefore, for any point $a \in \mathbf{R}^{2}$, there exists a nontrivial solution to (6.2) (6.3) passing through $a$.

\subsection{Bounds on mean curvature of surfaces}

Let $M$ be a compact surface in $\mathbf{R}^{3}$, diffeomorphic to $S^{2}$. Let $A$ denote the area of $M$, and $D$ its diameter, i.e.

$$
D(M)=\max _{x, y \in M}|x-y|
$$

We have the following. 
Theorem 6.2.- There exists some constant $C$, such that

$$
\max _{x \in M}|H(x)| \geq C \frac{D(M)}{A(M)} .
$$

Sketch of the proof. By a theorem of Sacks and Uhlenbeck [28], there is an harmonic conformal parametrization $u: S^{2} \rightarrow M$ of $M$. $u$ satisfies

$$
\Delta u=2 H(u) u_{x} \wedge u_{y}
$$

Then, from the estimates of Theorem 4.8-4.10, we deduce that

$$
\frac{1}{2} D(M) \leq\|u\|_{L^{\infty}} \leq C\|H\|_{L^{\infty}} \int_{S^{2}}|\nabla u|^{2}
$$

Indeed, (6.6) is a direct consequence of the previous theorems in the case $A(M)=1$, and the general case follows from a simple scaling argument.

Note that $C$ should be optimal in the case of a round sphere, i.e. $C=\frac{\pi}{2}$.

In the case where $M$ is not assumed to be diffeomorphic to $S^{2}$ any more, results of Sacks and Uhlenbeck [29] lead to a similar estimate, with a constant depending on the genus of the surface, i.e.

$$
\max _{x \in M}|H(x)| \geq C(\operatorname{genus}(M)) \frac{D(M)}{A(M)} .
$$

\subsection{Prescribing curvature in manifolds}

An interesting problem would be now to investigate surfaces of precribed mean curvature with values in a three dimensional manifold, instead of $\mathbf{R}^{3}$ - a question which is related to the isoperimetric problem. 


\section{APPENDIX}

\section{Behaviour of the Palais-Smale sequences}

In this appendix, we give the proof of Theorem 5.6. Throughout this section we assume that the function $H: \mathbf{R}^{3} \rightarrow \mathbf{R}$ satisfies the condition

$$
\sup _{u \in \mathbf{R}^{3}}(|H(u)|+(1+|u|)|\nabla H(u)|)<+\infty
$$

We consider sequences $\left(u^{n}\right) \in H^{1}\left(D^{2}, \mathbf{R}^{3}\right)$ verifying

$$
\Delta u^{n}=2 H\left(u^{n}\right) u_{x}^{n} \wedge u_{y}^{n}+f^{n} \quad \text { in } \quad D^{2}
$$

with

$$
\begin{gathered}
f^{n} \rightarrow 0 \text { in } H^{-1} \\
\left\|u^{n}\right\|_{H^{1}\left(D^{2}\right)} \leq C \\
\left\|u^{n}\right\|_{H^{1 / 2}\left(\partial D^{2}\right.}+\left\|u^{n}\right\|_{L^{\infty}\left(\partial D^{2}\right)} \leq C
\end{gathered}
$$

We assume furthermore that $\left(u^{n}\right)$ converges strongly in $H^{1 / 2}\left(\partial D^{2}\right)$ and uniformly, to some $\gamma \in\left(H^{1 / 2} \cap L^{\infty}\right)\left(\partial D^{2}\right)$.

Since $\left(u^{n}\right)$ is bounded in $H^{1 / 2}\left(D^{2}\right)$, we may assume that $\left(u^{n}\right)$ converges weakly in this space to a map $u^{0} .|\nabla H|$ being uniformly bounded in $\mathbf{R}^{3}$, as (A.1) ensures, if follows from a result of [3] that $u^{0}$ satisfies

$$
\begin{gathered}
\Delta u^{0}=2 H\left(u^{0}\right) u_{x}^{0} \wedge u_{y}^{0} \text { in } D^{2} \\
u^{0}=\gamma \text { on } \partial D^{2} .
\end{gathered}
$$

Moreover, by a result of Heinz (see also [2]), $u^{0}$ is smooth in $D^{2}$. Since $u^{0}$ is bounded on $\partial D^{2}$, we obtain, in view of Theorem 4.8 .

$$
\left\|u^{0}\right\|_{L^{\infty}\left(D^{2}\right)} \leq C,
$$

where $C$ is some constant.

Our aim is to give a precise description of the convergence of $\left(u^{n}\right)$ to $u^{0}$, and to show that the defect of strong convergence is due to the concentration of the energy at isolated points. This phenomenon has been observed in numerous other situations, starting from the pioneering work of Sacks and Uhlenbeck - see for instance BahriCoron [1], P.L. Lions [21], Brézis-Coron [8]. Here, we basically adapt the strategy of [8]. The main ingredient in the proof is the following: 
Lemma A.1. - Let $z_{0}$ be a point in $D^{2}$, and $0<r<\frac{1}{2}$. There is some constant $\varepsilon_{0}>0$ such that if

$$
\int_{B\left(z_{0}, 2 r\right) \cap D^{2}}\left|\nabla u^{n}\right|^{2} \leq \varepsilon_{0}
$$

for $n$ large enough, then $\left(u^{n}\right)$ converges strongly to $u^{0}$ in $B\left(z_{0}, r\right) \cap D^{2}$.

Proof of the lemma. The proof is divided in four steps.

Step 1. Given any $n$, there is some $\left.r^{n} \in\right] r, 2 r[$ such that

$$
\left.\left.u^{n}\right|_{\partial\left[B\left(z_{0}, r^{n}\right) \cap D^{2}\right]} \rightarrow u^{0}\right|_{\partial\left[B\left(z_{0}, r^{n}\right) \cap D^{2}\right]}
$$

strongly in $H^{1 / 2} \cap L^{\infty}$.

Proof of step 1. Since $\left(u^{n}\right)$ converges weakly in $H^{1}$ to $u^{0},\left(u^{n}\right)$ converges strongly in $L^{2}$. By Fubini's theorem, there is some $r^{n}$ in $] r, 2 r[$ such that

$$
\int_{\partial B\left(z_{0}, r^{n}\right) \cap D^{2}}\left|\nabla\left(u^{n}-u^{0}\right)\right|^{2} \leq 2 \frac{\varepsilon_{0}}{r}
$$

and

$$
\int_{\partial B\left(z_{0}, r^{n}\right) \cap D^{2}}\left|u^{n}-u^{0}\right|^{2} \rightarrow 0 \quad \text { as } \quad n \rightarrow+\infty .
$$

We have the interpolation inequality

$$
\begin{gathered}
\left\|u^{n}-u^{0}\right\|_{H^{1 / 2}\left(\partial B(z, r) \cap D^{2}\right)} \leq C\left\|u^{n}-u^{0}\right\|_{H^{1}\left(\partial B\left(z_{0}, r^{n}\right) \cap D^{2}\right)} \\
.\left\|u^{n}-u^{0}\right\|_{L^{2}\left(\partial B\left(z_{0}, r^{n}\right) \cap D^{2}\right)} .
\end{gathered}
$$

Therefore, combining (A.11) (A.12) and (A.13), we see that

$$
u^{n} \rightarrow u^{0} \quad \text { in } \quad H^{1 / 2}\left(\partial B\left(z_{0}, r^{n}\right) \cap D^{2}\right) .
$$

Similarly, (A.11) (A.12) and the Sobolev embedding show that

$$
u^{n} \rightarrow u^{0} \quad \text { uniformly in } \quad L^{\infty}\left(\partial B\left(z_{0}, r^{n}\right) \cap D^{2}\right)
$$

Since $u^{n} \rightarrow u^{0}$ in $H^{1 / 2} \cap L^{\infty}\left(\partial D^{2}\right),($ A.10) follows.

We set

$$
\begin{aligned}
& \Omega^{n}=B\left(z_{0}, r^{n}\right) \cap D^{2} \\
& T^{n}=\partial \Omega^{n}
\end{aligned}
$$


and

$$
M=\limsup _{n \rightarrow+\infty}\left\|u^{n}\right\|_{L^{\infty}\left(T^{n}\right)}
$$

It follows from (A.10) that

$$
M<+\infty
$$

For $k \in \mathbf{N}, k>M+1$, and $n \in \mathbf{N}^{*}$, we set also

$$
\begin{aligned}
W^{n}(k) & =\left\{x \in \Omega^{n} /\left|u^{n}(x)\right|>k\right\} \\
\Lambda^{n}(k) & =\int_{W^{n}(k)}\left|\nabla u^{n}\right|^{2}
\end{aligned}
$$

and

$$
\Lambda(k)=\limsup _{u \rightarrow+\infty} \Lambda^{n}(k)
$$

Step 2. We have

$$
\Lambda(k) \rightarrow 0 \quad \text { as } \quad k \rightarrow+\infty
$$

Proof of step 2. We adapt an argument of [4]. For $m \in \mathbf{N}$, let $\zeta_{m}$ be a smooth function from $\mathbf{R}^{+}$to $[0,1]$ such that

$$
\begin{gathered}
\zeta_{m}(t)=0 \quad \text { if } \quad t \leq 2^{m} \\
\zeta_{m}(t)=1 \quad \text { if } \quad t \geq 2^{m+1}
\end{gathered}
$$

and

$$
0 \leq t \zeta_{m}^{\prime}(t) \leq 2
$$

We set

$$
v_{m}^{n}=\zeta_{m}\left(\left|u^{n}\right|\right) u^{n}
$$

Note that $v_{m}^{n}(z)=0$ on $\partial \Omega^{n}$, if $2^{m}>M$ and $n$ is large enough, and that $v_{m}^{n}(z)=$ $u^{n}(z)$ if $\left|u^{n}(z)\right|>2^{m+1}$. Moreover, we easily verify that $v_{m}^{n}$ belongs to $H^{1}\left(D^{2}\right)$. Now, we multiply (A.2) by $v_{m}^{n}$ and integrate on $\Omega^{n}$. We obtain

$$
-\int_{\Omega^{n}} \nabla v_{m}^{n} \cdot \nabla u^{n}=\int_{\Omega^{n}} 2 H\left(u^{n}\right) v_{m}^{n} \cdot u_{x}^{n} \wedge u_{y}^{n}+\left(f^{n}, v_{m}^{n}\right)
$$

We have

$$
\int_{\Omega^{n}} \nabla v_{m}^{n} \cdot \nabla u^{n}=\int_{\Omega^{n}} \zeta_{m}\left(\left|u^{n}\right|\right)\left|\nabla u^{n}\right|^{2}+\int_{\Omega^{n}} \zeta_{m}^{\prime}\left(\left|u^{n}\right|\right)\left|u^{n}\right||\nabla| u^{n} \|^{2}
$$


so that, from the definition of $\Lambda^{n}$, and the fact that $\zeta_{m}^{\prime} \geq 0$

$$
\Lambda^{n}\left(2^{m+1}\right) \leq \int_{\Omega^{n}} \zeta_{m}\left(\left|u^{n}\right|\right)\left|\nabla u^{n}\right|^{2} \leq \int_{\Omega^{n}} \nabla v_{m}^{n} . \nabla u^{n}
$$

Since $\zeta_{m-1}\left(\left|u^{n}\right|\right)=0$ if $\left|u^{n}\right|<2^{m-1}$, and $\zeta_{m-1}\left(\left|u^{n}\right|\right)=1$ if $\left|u^{n}\right|>2^{m}$, we have

$$
H\left(u^{n}\right) v_{m}^{n} \cdot u_{x}^{n} \Lambda u_{y}^{n}=H\left(u^{n}\right) v_{m}^{n} \cdot\left(\zeta_{m-1}\left(\left|u^{n}\right|\right) u^{n}\right)_{x} \wedge\left(\zeta_{m-1}\left(\left|u^{n}\right|\right) u^{n}\right)_{y}
$$

In view of (A.1) and (A.20), we verify that

$$
\left|\nabla\left(H\left(u^{n}\right) v_{m}^{n}\right)\right| \leq C\left|\nabla u^{n}\right|
$$

and

$$
\left|\nabla\left(\zeta_{m-1}\left(\left|u^{n}\right|\right) u^{n}\right)\right| \leq C\left|\nabla u^{n}\right|
$$

where $C$ is some constant.

Let $\varphi^{n}$ be the solution of

$$
\left\{\begin{aligned}
\Delta \varphi^{n} & =\left(\zeta_{m-1}\left(\left|u^{n}\right|\right) u^{n}\right)_{x} \wedge\left(\zeta_{m-1}\left(\left|u^{n}\right|\right) u^{n}\right)_{y} \text { in } \Omega^{n} \\
\varphi^{n} & =0 \text { on } \partial \Omega^{n} .
\end{aligned}\right.
$$

By a result of Wente [43] we have, since the right hand side is a Jacobian

$$
\int_{\Omega^{n}}\left|\nabla \varphi^{n}\right|^{2} \leq C\left(\int_{\Omega^{n}}\left|\nabla\left(\zeta_{m-1}\left(\left|u^{n}\right|\right) u^{n}\right)\right|^{z}\right)^{2} .
$$

Hence, using (A.25)

$$
\int_{\Omega^{n}}\left|\nabla \varphi^{n}\right|^{2} \leq C\left(\int_{W^{n}\left(2^{m-1}\right)}\left|\nabla u^{n}\right|^{2}\right)^{2}
$$

that is

$$
\int_{\Omega^{n}}\left|\nabla \varphi^{n}\right|^{2} \leq C\left(\Lambda^{n}\left(2^{m-1}\right)\right)^{2}
$$


We write, in view of (A.23)

$$
\begin{aligned}
& \left|\int_{\Omega^{n}} H\left(u^{n}\right) v_{m}^{n} \cdot u_{x}^{n} \wedge u_{y}^{n}\right| \\
& =\left|\int_{\Omega^{n}} H\left(u^{n}\right) v_{m}^{n} \cdot\left(\zeta_{m-1}\left(\left|u^{n}\right|\right) u^{n}\right)_{x} \wedge\left(\zeta_{m-1}\left(\left|u^{n}\right|\right) u^{n}\right)_{y}\right| \\
& =\left|\int_{\Omega^{n}} H\left(u^{n}\right) v_{m}^{n} \cdot \Delta \varphi^{n}\right| \\
& =\left|\int_{\Omega^{n}} \nabla\left(H\left(u^{n}\right) v_{m}^{n}\right) \cdot \nabla \varphi^{n}\right| \\
& \leq \int_{\Omega^{n}}\left|\nabla\left(H\left(u^{n}\right) v_{m}^{n}\right)\right|\left|\nabla \varphi^{n}\right| \\
& \leq C \int_{W^{n}\left(2^{m}\right)}\left|\nabla u^{n}\right|\left|\nabla \varphi^{n}\right| \quad \text { by }(A .24) \\
& \leq C\left(\int_{W^{n}\left(2^{m}\right)}\left|\nabla u^{n}\right|^{2}\right)^{1 / 2}\left(\int_{\Omega^{n}}\left|\nabla \varphi^{n}\right|^{2}\right)^{1 / 2} \\
& \leq C\left(\Lambda^{n}\left(2^{m}\right)\right)^{1 / 2} \Lambda^{n}\left(2^{m-1}\right) \quad \text { by }(A .26) .
\end{aligned}
$$

Finally, we obtain

$$
\left|\int_{\Omega^{n}} H\left(u^{n}\right) v_{m}^{n} \cdot u_{x}^{n} \wedge u_{y}^{n}\right| \leq C\left(\Lambda^{n}\left(2^{m-1}\right)\right)^{3 / 2} .
$$

On the other hand, $v_{m}^{n}$ being bounded in $H^{1}$, and $f^{n}$ going to zero in $H^{-1}$, we have

$$
\left(f^{n}, v_{m}^{n}\right) \rightarrow 0 \quad \text { as } n \rightarrow+\infty .
$$

Combining (A.21) (A.22) (A.27) and (A.28), we obtain

$$
\Lambda^{n}\left(2^{m+1}\right) \leq C\left(\Lambda^{n}\left(2^{m-1}\right)\right)^{3 / 2}+o(1) \quad \text { as } n \rightarrow+\infty .
$$

By (A.9), we have

$$
\Lambda^{n}(k) \leq \varepsilon_{0} \quad \text { for any } k, \text { and any } n \text { large enough }
$$

so that, iterating (A.29), we obtain

$$
\Lambda^{n}\left(2^{m}\right) \leq C \varepsilon_{0}^{m / 2}+o(1) \text { as } n \rightarrow+\infty .
$$

We first let $n$ go to infinity, then $m$, and (A.17) follows (one can assume that $\varepsilon_{0}<1$ ).

Next, we set, for $k>M+1$

$$
\begin{aligned}
& F^{n}(k)=\left\{z \in \Omega^{n} /\left|u^{n}(z)\right| \leq k\right\} \\
& A^{n}(k)=\int_{F^{n}(k)}\left|\nabla\left(u^{n}-u^{0}\right)\right|^{2}
\end{aligned}
$$


and

$$
A(k)=\lim \inf _{n \rightarrow+\infty} A^{n}(k) .
$$

Step3. We have

$$
A(k) \rightarrow 0 \quad \text { as } \quad k \rightarrow+\infty
$$

Proof of step 3. For $m \in \mathbf{N}$, let $\xi_{m}$ be a smooth map from $\mathbf{R}$ to $[0,1]$ such that

$$
\xi_{m}(t)=1 \quad \text { if } \quad t<2^{m}
$$

$$
\xi_{m}(t)=0 \quad \text { if } \quad t>2^{m+1}
$$

and

$$
-2 \leq t \xi_{m}^{\prime}(t) \leq 0
$$

Let $\psi^{n}$ be the solution of

$$
\left\{\begin{aligned}
\Delta \psi^{n} & =0 \text { in } \Omega^{n} \\
\psi^{n} & =u^{n}-u^{0} \quad \text { on } \partial \Omega^{n} .
\end{aligned}\right.
$$

Since, according to step $1, u^{n}$ converges strongly to $u^{0}$ in $H^{1 / 2}\left(T^{n}\right)$ and uniformly on this boundary, we have

$$
\psi^{n} \rightarrow 0 \text { in } H^{1} \cap L^{\infty}\left(\Omega^{n}\right)
$$

Set

$$
u^{n, 0}=u^{0}+\psi^{n},
$$

so that

$$
u^{n, 0} \rightarrow u^{0} \quad \text { in } \quad H^{1} \cap L^{\infty}\left(\Omega^{n}\right)
$$

and

$$
\left\{\begin{aligned}
\Delta u^{n, 0} & =2 H\left(u^{0}\right) u_{x}^{0} \wedge u_{y}^{0} \quad \text { in } \quad \Omega^{n} \\
u^{n, 0} & =0 \text { on } \partial \Omega^{n} .
\end{aligned}\right.
$$

We consider the function $w_{m}^{n}$ defined, for $m \in \mathbf{N}$, by

$$
w_{m}^{n}=\xi_{m}\left(\left|u^{n}\right|\right)\left(u^{n}-u^{n, 0}\right) .
$$


Note that $\left\|w_{m}^{n}\right\|_{L^{\infty}} \leq 2^{m+1}+M$ for $n$ large enough. We use $w_{m}^{n}$ as a test function in (A.2) (A.36), i.e. we multiply these two equations by $w_{m}^{n}$, and integrate on $\Omega^{n}$. Substracting the second equality to the first one, we obtain

$$
\begin{gathered}
-\int_{\Omega^{n}} \nabla w_{m}^{n} \cdot \nabla\left(u^{n}-u^{n, 0}\right) \\
=2 \int_{\Omega^{n}} H\left(u^{n}\right) w_{m}^{n} \cdot u_{x}^{n} \wedge u_{y}^{n}-2 \int_{\Omega^{n}} H\left(u^{0}\right) w_{m}^{n} \cdot u_{x}^{0} \wedge u_{y}^{0}+\left(f^{n}, w_{m}^{n}\right) .
\end{gathered}
$$

We write

$$
-\int_{\Omega^{n}} \nabla w_{m}^{n} \cdot \nabla\left(u^{n}-u^{n, 0}\right)=I_{1}^{n}+I_{2}^{n}+I_{3}^{n}+I_{4}^{n}
$$

with

and

$$
\begin{aligned}
& I_{1}^{n}=2 \int_{\Omega^{n}} H\left(u^{n}\right) w_{m}^{n} \cdot\left(u^{n}-u^{0}\right)_{x} \wedge\left(u^{n}-u^{0}\right)_{y} \\
& I_{2}^{n}=-4 \int_{\Omega^{n}} H\left(u^{n}\right) w_{m}^{n} \cdot\left(u_{x}^{0} \wedge u_{y}^{n}+u_{x}^{n} \wedge u_{y}^{0}\right) \\
& I_{3}^{n}=2 \int_{\Omega^{n}}\left(H\left(u^{n}\right)-H\left(u^{0}\right)\right) w_{m}^{n} \cdot u_{x}^{0} \wedge u_{y}^{0} \\
& I_{4}^{n}=\left(f^{n}, w_{m}^{n}\right) .
\end{aligned}
$$

We fix $m$ for the moment, and let $n$ go to infinity.

Since $f^{n} \rightarrow 0$ in $H^{-1}$ as $n \rightarrow+\infty$, we have

$$
I_{4}^{n} \rightarrow 0 \quad \text { as } n \rightarrow+\infty \text {. }
$$

$u^{n}-u^{n, 0}$ converging weakly to zero in $H^{1}, u^{n}-u^{n, 0}$ converges to zero almost everywhere, and therefore

$$
w_{m}^{n} \rightarrow 0 \text { almost everywhere . }
$$

On the other hand, $w_{m}^{n}$ is uniformly bounded. Hence we may apply Lebesgue's theorem, which yields

$$
I_{2}^{n} \rightarrow 0 \quad \text { as } \quad n \rightarrow+\infty
$$

and

$$
I_{3}^{n} \rightarrow 0 \quad \text { as } \quad n \rightarrow+\infty \text {. }
$$

We turn now to $I_{1}^{n}$. Let $\Phi^{n}$ be the solution of

$$
\left\{\begin{aligned}
\Delta \Phi^{n} & =\left(u^{n}-u^{0}\right)_{x} \wedge\left(u^{n}-u^{0}\right)_{y} \text { in } \Omega^{n} \\
\Phi^{n} & =0 \text { on } \partial \Omega^{n}
\end{aligned}\right.
$$


By the above quoted result of Wente, we have

$$
\int_{\Omega}\left|\nabla \Phi^{n}\right|^{2} \leq\left(\int_{\Omega^{n}}\left|\nabla\left(u^{n}-u^{0}\right)\right|^{2}\right)^{2}
$$

We write

$$
\begin{aligned}
\left|I_{1}^{n}\right| & =\left|\int_{\Omega^{n}} 2 H\left(u^{n}\right) w_{m}^{n} \cdot \Delta \Phi^{n}\right| \\
& =2\left|\int_{\Omega^{n}} \nabla\left(H\left(u^{n}\right) w_{m}^{n}\right) \cdot \nabla \Phi^{n}\right| \\
& \leq 2\left(\int_{\Omega^{n}} \mid \nabla\left(\left.H\left(u^{n}\right) w_{m}^{n}\right|^{2}\right)^{1 / 2}\left(\int_{\Omega^{n}}\left|\nabla \Phi^{n}\right|^{2}\right)^{1 / 2}\right. \\
& \leq C\left(\int_{\Omega^{n}}\left(\left|\nabla u^{n}\right|^{2}+\left|\nabla u^{0}\right|^{2}\right)\right)^{1 / 2} \int_{\Omega}\left|\nabla\left(u^{n}-u^{0}\right)\right|^{2},
\end{aligned}
$$

where we have used (A.41) and the facts that

$$
\begin{gathered}
\left|\nabla w_{m}^{n}\right| \leq C\left(\left|\nabla u^{n}\right|+\left|\nabla u^{0}\right|\right) \\
\left|\nabla\left(H\left(u^{n}\right) w_{m}^{n}\right)\right| \leq C\left(\left|\nabla u^{n}\right|+\left|\nabla u^{0}\right|\right) .
\end{gathered}
$$

Note that the last inequality strongly relies on assumption (A.1).

By (A.9) we are led to

$$
\left|I_{1}^{n}\right| \leq C \varepsilon_{0}^{1 / 2} \int_{\Omega^{n}}\left|\nabla\left(u^{n}-u^{0}\right)\right|^{2} .
$$

Hence, we see that the right hand side of (A.37) may be estimated by

$$
\left|I_{1}^{n}+I_{2}^{n}+I_{3}^{n}+I_{4}^{n}\right| \leq C \varepsilon_{0}^{1 / 2} \int_{\Omega^{n}}\left|\nabla\left(u^{n}-u^{0}\right)\right|^{2}+o(1)
$$

as $n \rightarrow+\infty$

We handle next the right hand side of (A.37). We have

$$
\begin{gathered}
\int_{\Omega^{n}} \nabla w_{m}^{n} \nabla\left(u^{n}-u^{n, 0}\right)=\int_{\Omega^{n}} \xi_{m}\left(\left|u^{n}\right|\right)\left|\nabla\left(u^{n}-u^{n, 0}\right)\right|^{2} \\
+\int_{\Omega^{n}} \xi_{m}^{\prime}\left(\left|u^{n}\right|\right)\left(\nabla\left|u^{n}\right|\right)\left|u^{n}-u^{n, 0}\right|\left(\nabla\left|u^{n}-u^{n, 0}\right|\right)
\end{gathered}
$$

From the definition of $\xi_{m}$ and the boundedness of $u^{n, 0}$ in $L^{\infty}$, which follows from (A.8) and (A.35), we deduce that

$$
\left|\int_{\Omega^{n}} \xi_{m}^{\prime}\left(\left|u^{n}\right|\right)\left(\nabla\left|u^{n}\right|\right)\right| u^{n}-u^{n, 0}\left|\left(\nabla\left|u^{n}-u^{n, 0}\right|\right)\right|
$$




$$
\leq C\left(\int_{W^{n}\left(2^{m}\right)}\left|\nabla u^{n}\right|^{2}\right)^{1 / 2}\left(\int_{W^{n}\left(2^{m}\right)}\left|\nabla\left(u^{n}-u^{n, 0}\right)\right|^{2}\right)^{1 / 2} .
$$

On the other hand, we easily verify that

$$
\int_{\Omega^{n}} \xi_{m}\left(\left|u^{n}\right|\right)\left|\nabla\left(u^{n}-u^{n, 0}\right)\right|^{2} \geq \int_{F^{n}\left(2^{m}\right)}\left|\nabla\left(u^{n}-u^{n, 0}\right)\right|^{2}=A^{n}\left(2^{m}\right) .
$$

Combining (A.37) (A.44) (A.45) (A.46) and (A.47), we obtain

$$
\begin{aligned}
A^{n}\left(2^{m}\right) \leq C & \left(\int_{W^{n}\left(2^{m}\right)}\left|\nabla u^{n}\right|^{2}\right)^{1 / 2}\left(\int_{W^{n}\left(2^{m}\right)}\left|\nabla\left(u^{n}-u^{n, 0}\right)\right|^{2}\right)^{1 / 2} \\
& +C \varepsilon_{0}^{1 / 2} \int_{\Omega^{n}}\left|\nabla\left(u^{n}-u^{0}\right)\right|^{2}+o(1),
\end{aligned}
$$

as $n \rightarrow+\infty$. Note that $\left(u^{n}\right)$ and $\left(u^{n, 0}\right)$ being bounded in $H^{1}$, the first term in the right hand side is less than $C \Lambda^{n}\left(2^{m}\right)$. On the other hand, we claim that

$$
\int_{\Omega^{n}}\left|\nabla\left(u^{n}-u^{0}\right)\right|^{2}=A^{n}(k)+\Lambda^{n}(k)+o(1)
$$

as $k \rightarrow+\infty$. Indeed

$$
\begin{gathered}
\int_{\Omega^{n}}\left|\nabla\left(u^{n}-u^{0}\right)\right|^{2}=\int_{W^{n}(k)}\left|\nabla\left(u^{n}-u^{0}\right)\right|^{2}+\int_{F^{n}(k)}\left|\nabla\left(u^{n}-u^{0}\right)\right|^{2} \\
=A^{n}(k)+\Lambda^{n}(k)+\int_{W^{n}(k)}\left|\nabla u^{0}\right|^{2}-2 \nabla u^{n} \cdot \nabla u^{0}
\end{gathered}
$$

and

$$
\int_{W^{n}(k)}\left|\nabla u^{0}\right|^{2} \rightarrow 0 \quad \text { as } \quad k \rightarrow+\infty
$$

since $u^{0} \in H^{1}$ and mes $\left(W^{n}(k)\right) \rightarrow 0$ as $k \rightarrow+\infty$.

Choosing $\varepsilon_{0}$ such that $C \varepsilon_{0}^{1 / 2}<1$, we deduce from (A.48) the inequality

$$
A^{n}\left(2^{m}\right) \leq C \Lambda^{n}\left(2^{m}\right)+o(1) .
$$

as $n, k \rightarrow+\infty$. (A.50), combined with (A.17), yields (A.31)

Step 4. We are now able to complete the proof of Lemma 1.1. We write, for $k>M$

$$
\int_{\Omega^{n}}\left|\nabla\left(u^{n}-u^{n, 0}\right)\right|^{2}=A^{n}(k)+\Lambda^{n}(k)+o(1)
$$


by (A.49) and (A.35). Passing to the limit $n \rightarrow+\infty, k \rightarrow+\infty$, a standard diagonal argument shows that, up to a subsequence, by steps 2 and 3

$$
\int_{\Omega^{n}}\left|\nabla\left(u^{n}-u^{n, 0}\right)\right|^{2} \rightarrow 0 \quad \text { as } n \rightarrow+\infty .
$$

Since $u^{n, 0} \rightarrow u^{0}$ strongly in $H^{1}$, we obtain

$$
\int_{\Omega^{n}}\left|\nabla\left(u^{n}-u^{0}\right)\right|^{2} \rightarrow 0 \quad \text { as } n \rightarrow+\infty .
$$

Hence, since $\left(B\left(z_{0}, r\right) \cap D^{2}\right) \subset \Omega^{n}$,

$$
\int_{B\left(z_{0}, r\right) \cap D^{2}}\left|\nabla\left(u^{n}-u^{0}\right)\right|^{2} \rightarrow 0 .
$$

In the course of the proof, we have extracted many subsequences. However, in order to prove that (A.51) holds for the full sequence, we may argue by contradiction, and assume that for some subsequence $n^{\prime} \rightarrow+\infty$ we have

$$
\int_{B\left(z_{0}, r\right) \cap D^{2}}\left|\nabla\left(u^{n^{\prime}}-u^{0}\right)\right|^{2} \geq \mu
$$

for some $\mu>0$. Repeating our arguments, (A.51) would hold for a subsequence $\left(u^{n^{\prime \prime}}\right)$ of $\left(u^{n^{\prime}}\right)$, a contradiction. This completes the proof of the lemma.

Proof of Theorem 5.6 completed.

For $r>0$, let $\left(B\left(z_{i}^{r}, \frac{r}{2}\right)\right)_{1 \leq i \leq \ell}$ be a maximal family of disjoint balls such that the balls $\left(B\left(z_{i}^{r}, r\right)\right)_{1 \leq i \leq \ell}$ cover $D^{2}$. Set

$$
J^{r}=\left\{i \in\{1, \cdots, \ell\} / \liminf _{n \rightarrow+\infty} \int_{B\left(z_{i}^{r}, 2 r\right)}\left|\nabla u^{n}\right|^{2}>\varepsilon\right\}
$$

In view of (A.4), there is some $N \in \mathbf{N}$ such that for any $r, 0<r<1$

$$
N^{r}=\sharp J^{r}<N \text {. }
$$

Hence, passing to a subsequence $r_{k} \underset{k \rightarrow+\infty}{\rightarrow} 0$, we may assume that

$$
N^{r_{k}}=\bar{N}
$$

and, relabelling the points if necessary, that

$$
\begin{gathered}
J^{r_{k}}=\{1, \cdots, \bar{N}\} \\
z_{i}^{r_{k}} \rightarrow a_{i} \quad \forall i, 1 \leq i \leq \bar{N}
\end{gathered}
$$

where the $a_{i}^{\prime} s$ are points in $\bar{D}^{2}$. Lemma A.1 ensures that up to a subsequence, for any compact subset $\Omega$ of $\bar{D}^{2} \backslash \cup_{1 \leq i \leq \bar{N}}\left\{a_{i}\right\}$

$$
u^{n} \rightarrow u^{0} \text { in } H^{1}(\Omega) \text {. }
$$

This proves assertion (i) of the theorem.

Assertion (ii) (iii) (iv) then follow from a standard blow-up analysis at the points $a_{i}$, and may be proved exactly as performed in [8], in the case $H \equiv$ constant. 


\section{REFERENCES}

[1] A. Bahri, J.-M. Coron - On a nonlinear elliptic equation involving the critical Sobolev exponent : the effect of the topology of the domain, Comm. Pure Appl. Mat. 41 (1988), 255-294.

[2] F. Bethuel - Un résultat de régularité pour les solutions de l'équation des surfaces à courbure moyenne prescrite, C. R. Acad. Sci. Paris 314 (1992), 1003-1007.

[3] F. Bethuel - Weak convergence of Palais-Smale sequences for some critical functionals, to appear.

[4] F. Bethuel, J.-M. Ghidaglia - Regularity for solutions to the equation of surfaces of prescribed mean curvature using the coarea formula, to appear.

[5] F. Bethuel, O. Rey - Large solutions for the equation of surfaces of prescribed mean curvature, Geometry in PDE's, T.M. Rassias and A. Prastaro ed., World Scientific Publishing Co. (1993).

[6] F. Bethuel, O. Rey - Le problème des surfaces à courbure moyenne prescrite, Séminaire EDP de l'Ecole Polytechnique, Exposé V (1992).

[7] H. Brezis, J.-M. Coron - Multiple solutions of H-Systems and Rellich's conjecture, Comm. Pure Appl. Math. 37 (1984), 149-187.

[8] H. Brezis, J.-M. Coron - Convergence of solutions of H-Systems or how to blow bubbles, Arch. Rat. Mech. Anal. 89 (1985), 21-56.

[9] S.S. Chern, S.I. Goldberg - On the volume-decreasing property of a class of real harmonic mappings, Ann. J. Math. 97 (1975), 133-147.

[10] J. Douglas - Solutions of the Problem of Plateau, Trans. Amer. Math. Soc. 33 (1983), 263-321.

[11] F.W. Gehring - The $L^{p}$-integrability of the partial derivatives of a quasi conformal mapping, Acta Math. 130 (1973), 265-277.

[12] M. Giaquinta - Multiple Integrals in the Calculus of Variations and Nonlinear Elliptic Systems, Princeton University Press (1983).

[13] M. Grüter - Regularity of weak H-Surfaces, J. Reine Angew. Math. 329 (1981), $1-15$.

[14] M. Grüter - Eine Bemerkung zu Regularität stationärer Punkte von konform invarianten Variationsintegralen, Manuscripta Math. 55 (1986), 451-453. 
[15] E. Heinz - Über die Existenz einer Fläche konstanter mittlerer Krümmung bei vorgegebener Berandung, Math. Ann. 127 (1954), 258-287.

[16] E. Heinz - On the non-existence of a surface of constant mean curvature with finite area and prescribed rectifiable boundary, Arch. Rat. Mech. Anal. 35 (1969), 249-252.

[17] S. Hildebrandt - Randwertprobleme für Flächen mit vorgeschriebener mittlerer Krümmung und Anwendungen auf die Kapillaritätstheorie, Math. Z. 112 (1969), 205-213.

[18] S. Hildebrandt - On the Plateau problem for surfaces of constant mean curvature, Comm. Pure Appl. Math. 23 (1970), 97-114.

[19] D. Hoffmann, J. Spruck - Sobolev and isoporimetric inequalities for Riemannian submanifolds, Comm. Pure Appl. Math. 27 (1974), 715-727, and 28 (1975), 765-766.

[20] L. Lemaire - Applications harmoniques des surfaces Riemanniennes, Thèse, Université Libre de Bruxelles (1975).

[21] P.L. Lions - The concentration compactness principle in the calculus of variations, the limit case, Rev. Mat. Iberoamericana $\mathbf{1 . 1}$ (1985), 145-201, and $\mathbf{1 . 2}$ (1985), 45-121.

[22] C. Morrey - Multiple integrals in the calculus of variations, Springer, Berlin (1966).

[23] F. Pacard - Convergence of surfaces of prescribed mean curvature, J. Nonlinear Anal. TMS 13 (1989), 1269-1281.

[24] T. Radó - On Plateau's problem, Ann. of Math. 31 (1930), 457-469.

[25] T. Radó - The problem of least area and the problem of Plateau, Math. Z. 32 (1930), 763-796.

[26] T. Radó - Contributions to the theory of minimal surfaces, Act. Litt. Sci. Univ. Szeged 6 (1932), 1-20.

[27] O. Rey - Heat flow for the equation of surfaces with prescribed mean curvature Math. Ann. 291 (1991), 123-146.

[28] J. Sacks, K. Uhlenbeck - The existence of minimal immersions of spheres, Ann. Math. 113 (1981), 1-24. 
[29] J. Sacks, K. Uhlenbeck - Minimal immersions of closed Riemann surfaces, Trans. Amer. Math. Soc. 271 (1982), 639-652.

[30] Y. Sasahara - Asymptotic Behavior of Large Solutions for H-systems, to appear.

[31] R. Schoen - Analytic aspects of the harmonic map problem, in Seminar on Nonlinear Partial Differential Equations, S.S. Chern ed., MSRI Publications 2, Springer (1984).

[32] K. Steffen - On the existence of surfaces with prescribed mean curvature and boundary, Math. Z. 146 (1976), 113-135.

[33] K. Steffen - On the nonuniqueness of surfaces with prescribed mean curvature spanning a given contour, Arch. Rat. Mech. Anal. 94 (1986), 101-122.

[34] M. Struwe - Nonuniqueness in the Plateau problem for surfaces of constant mean curvature, Arch. Rat. Mech. Anal. 93 (1986), 135-157.

[35] M. Struwe - Large H-surfaces via the mountain-pass Lemma, Math. Ann. 270 (1985), 441-459.

[36] M. Struwe - Plateau's problem and the Calculus of Variations, Mathematical Notes 35, Princeton University Press (1988).

[37] M. Struwe - Multiple solutions to the Dirichlet problem for the equation of prescribed mean curvature, Analysis, et caetera, Academic Press, Boston (1990).

[38] F. Tomi - Ein einfacher Beweis eines Regularitätssatzes für schwache Lösungen gewisser elliptischer Systeme, Math. Z. 112 (1969), 214-218.

[39] G. Wang - The Dirichlet problem for the equation of prescribed mean curvature, Ann. Inst. Henri Poincaré, Anal. non lineaire 9 (1992), 643-655.

[40] H. Wente - An existence theorem for surfaces of constant mean curvature, J. Math. Analysis Appl. 26 (1969), 318-344.

[41] H. Wente - A general existence theorem for surfaces of constant mean curvature, Math. Z. 120 (1971), 277-278.

[42] H. Wente - The differential equation $\Delta x=2 H\left(x_{u} \wedge x_{v}\right)$ with vanishing boundary values, Proc. A.M.S. 50 (1975), 113-137.

[43] H.Werner - Das Problem von Douglas fur Flächen konstanter mittlerer Krümmung Math. Ann. 133 (1957), 303-319. 